\title{
UV Capabilities to Probe the Formation of Planetary Systems: From the ISM to Planets
}

\author{
Ana I. Gómez de Castro • Alain Lecavelier · \\ Miguel D'Avillez · Jeffrey L. Linsky · José Cernicharo
}

Received: 26 April 2005 / Accepted: 16 June 2005

(C) Springer Science + Business Media B.V. 2006

\begin{abstract}
Planetary systems are angular momentum reservoirs generated during star formation. Solutions to three of the most important problems in contemporary astrophysics are needed to understand the entire process of planetary system formation:
\end{abstract}

The physics of the ISM. Stars form from dense molecular clouds that contain $\sim 30 \%$ of the total interstellar medium (ISM) mass. The structure, properties and lifetimes of molecular clouds are determined by the overall dynamics and evolution of a very complex system - the ISM. Understanding the physics of the ISM is of prime importance not only for Galactic but also for extragalactic and cosmological studies. Most of the ISM volume ( $\sim 65 \%)$ is filled with diffuse gas at temperatures between 3000 and $300000 \mathrm{~K}$, representing about $50 \%$ of the ISM mass.

The physics of accretion and outflow. Powerful outflows are known to regulate angular momentum transport dur-

A. I. G. de Castro

Instituto de Astronomía y Geodesia (CSIC-UCM), Universidad

Complutense de Madrid, Madrid E-28040, Spain

A. Lecavelier

Institute d'Astrophysique de Paris, Paris, France

M. D'Avillez

Department of Mathematics, University of Évora R. Romão Ramalho 59, 7000 Évora, Portugal; Institut f'ur Astronomie, Universität Wien, Türkenschanzstr. 17, A-1180 Wien, Austria

J. L. Linsky

JILA, University of Colorado and NIST, Boulder, CO

80309-0440, USA

J. Cernicharo

DAMIR-IEM-CSIC, C/. Serrano 113 \& 121, 28006 Madrid, Spain ing star formation, the so-called accretion-outflow engine. Elementary physical considerations show that, to be efficient, the acceleration region for the outflows must be located close to the star (within $1 \mathrm{AU}$ ) where the gravitational field is strong. According to recent numerical simulations, this is also the region where terrestrial planets could form after 1 Myr. One should keep in mind that today the only evidence for life in the Universe comes from a planet located in this inner disk region (at $1 \mathrm{AU}$ ) from its parent star. The temperature of the accretion-outflow engine is between 3000 and $10^{7} \mathrm{~K}$. After $1 \mathrm{Myr}$, during the classical $T$ Tauri stage, extinction is small and the engine becomes naked and can be observed at ultraviolet wavelengths.

The physics of planet formation. Observations of volatiles released by dust, planetesimals and comets provide an extremely powerful tool for determining the relative abundances of the vaporizing species and for studying the photochemical and physical processes acting in the inner parts of young planetary systems. This region is illuminated by the strong UV radiation field produced by the star and the accretion-outflow engine. Absorption spectroscopy provides the most sensitive tool for determining the properties of the circumstellar gas as well as the characteristics of the atmospheres of the inner planets transiting the stellar disk. UV radiation also pumps the electronic transitions of the most abundant molecules $\left(\mathrm{H}_{2}, \mathrm{CO}\right.$, etc. $)$ that are observed in the UV.

Here we argue that access to the UV spectral range is essential for making progress in this field, since the resonance lines of the most abundant atoms and ions at temperatures between 3000 and $300000 \mathrm{~K}$, together with the electronic transitions of the most abundant molecules $\left(\mathrm{H}_{2}, \mathrm{CO}, \mathrm{OH}, \mathrm{CS}\right.$, 
$\mathrm{S}_{2}, \mathrm{CO}_{2}^{+}, \mathrm{C}_{2}, \mathrm{O}_{2}, \mathrm{O}_{3}$, etc.) are at $\mathrm{UV}$ wavelengths. A powerful UV-optical instrument would provide an efficient mean for measuring the abundance of ozone in the atmosphere of the thousands of transiting planets expected to be detected by the next space missions (GAIA, Corot, Kepler, etc.). Thus, a follow-up UV mission would be optimal for identifying Earth-like candidates.

Keywords UV astronomy · ISM - Pre-main sequence stars $\cdot$ Jets $\cdot$ Winds $\cdot$ Accretion disks $\cdot$ Planets

\section{Introduction}

The formation of planetary systems covers a broad range of physical and astrophysical processes ranging from the physics of star formation (the interstellar medium (ISM), molecular clouds, and initial mass function), to the physics of accretion and outflow (accretion disk properties, winds generation, and disk instabilities) and finally, the formation of planets (dust nucleation, planetesimal and planet formation, planetary differentiation, planetary atmospheres and sustainable biological systems). The objective of this article is to summarize the reasons why access to the UV range is an essential requirement for making progress in these critically important areas of astrophysics.

For this reason, the article has been split into three key sections: physics of the ISM, physics of accretion and outflow, and planets and bio-markers. A summary has been added to the end of this contribution with the required UV capabilities to make progress in the field.

\section{The physics of the ISM}

Understanding the physics of the ISM is of prime importance not only for Galactic but also for extragalactic and cosmological studies. The ISM is everything observable in the Galaxy except for stars, e.g., gas (ionised, atomic and molecular), dust, high-energy particles (e.g., cosmic rays) and magnetic fields. The ISM is a very complex, highly non-linear dynamical system whose evolution controls star formation, gas mixing, and, therefore, the chemical enrichment of the Universe.

The ISM is often classified into five components: Hot Ionised Medium (HIM), Warm Ionised Medium (WIM), Warm Neutral Medium (WNM), Cold Neutral Medium (CNM) and dense Molecular Medium (MM) (see, e.g., Kulkarni and Heiles, 1988); the diffuse components (HIM, WIM, WHM and CNM) appear to be in approximate pressure equilibrium. The main properties of these components are summarized in Table 1. X-ray observations are most sensitive to the very hot gas with temperatures $T \geq 10^{6} \mathrm{~K}$. IR and radio wavelength observations are the only tools for studying the dense molecular gas where stars form. UV spectroscopy is the most sensitive tool for measuring the properties (column densities, temperatures, ionisation fractions, metallicity, depletions, etc.) of the diffuse gas in the 3000-300 $000 \mathrm{~K}$ temperature range, the WNM and WIM.

In the last few years, old models, based on pressure equilibrium between the various ISM phases (e.g., McKee and Ostriker, 1977), have been replaced by detailed numerical simulations that allow studying the ISM as it is, a dynamical system. High-spatial resolution numerical simulations now permit addressing a set of problems simultaneously, encompassing both large and small scales, provided that the appropriate grid size, resolution and numerical tools (e.g., adaptive mesh refinement) are used. Among the most important of these problems are: (i) global modelling to yield information on the formation and lifetimes of molecular clouds, (ii) how star-forming regions are influenced by large-scale flows in the ISM, and (iii) the dynamic roles that $\mathrm{SNe}$ and superbubbles play in triggering local and global star formation (see Heyer and Zweibel, 2004).

Key problems in ISM physics include the determination of the relative contributions to the energy input from the various possible sources ( $\mathrm{SNe}$, massive star winds and radiation fields, mass infall from the halo, galactic dynamics, cosmic rays and magnetic fields) and the roles of MHD turbulence and shocks in the energy cascade and structure formation. During the last few years, a very efficient feedback loop has been operating between radio observations and numerical simulations to study the role of MHD turbulence in the energy cascade within the densest regions of the ISM (H I and molecular clouds). A similar feedback loop needs to be established with UV observations to understand the heating/cooling processes and the overall ISM evolution, including the formation of molecular clouds.

Numerical simulations predict that $\sim 65 \%$ of the ISM volume (within the disk: $|z|<250 \mathrm{pc}$ ) is filled with gas at temperatures between $10^{3}$ and $10^{5.5} \mathrm{~K}$; in particular, the WIM $\left(10^{4}<T<10^{5.5} \mathrm{~K}\right)$ is expected to fill $25 \%$ of the disk volume, while the HIM filling factor is smaller (17\%) because it escapes to the halo (de Avillez and Breitschwerdt, 2004). These predictions agree with recent observations. The Wisconsin H-Alpha Mapper (WHAM) has observed O III emission extending to Galactic latitudes as high as $|b| \sim 45^{\circ}$, and even He I (5876 ̊) emission has been detected. However, $\mathrm{X}$-ray and extreme UV observations now show that the filling factor of the HIM in the local ISM is small. Clearly, UV instruments are required to make progress in our understanding of the physics of the Galactic ISM. This physics can be studied at two scales:

Large scale (>kpc scale): At this scale, the prime objectives are understanding the overall star formation efficiency and which parameters control the disk-halo circulation (the Galactic fountain). The roles of supernova 
Table 1 Components of the ISM (reference properties)

\begin{tabular}{lclll}
\hline Component & $n\left(\mathrm{~cm}^{-3}\right)$ & $T(\mathrm{~K})$ & Ionisation fraction & Spectral range \\
\hline HIM & Few $10^{-3}$ & $(5-10) \times 10^{6}$ & 1 & X-ray, UV \\
WIM ${ }^{\mathrm{a}, \mathrm{b}}$ & Few $10^{-1}$ & $10^{4}$ & 1 & UV, radio, optical \\
WNM & $1-10$ & $(3-8) \times 10^{3}$ & 0.1 & UV, optical, IR, radio \\
CNM & $1-50$ & $20-100$ & $10^{-2}$ & UV, IR, radio \\
MM & $10^{3}-10^{6}$ & 10 & $<10^{-4}$ & IR, radio \\
\hline
\end{tabular}

${ }^{a}$ In the last few years, a new sub-classification has been introduced to distinguish between the WIM envelope around molecular clouds, the so-called McKee \& Ostricker WIM or MOWIM, and the other warm, ionised components, the so-called Reynolds WIM.

${ }^{\mathrm{b}}$ This nominal temperature is often assigned because most of the observations come from $\mathrm{H} \alpha$ emission. However, UV observations have pointed out the presence of significantly hotter gas traced by $\mathrm{C}$ IV or O VI

explosions, expanding $\mathrm{H}$ II regions and the overall Galactic dynamics (shear, spiral arm shocks, high velocity cloud shocks, etc.) is examined in detail. Some attention is also devoted to understanding Galactic magnetic fields and the Galactic dynamo.

Small scale (the Local Bubble scale): The Local Bubble represents the nearest region of the ISM and is thus an ideal laboratory to test the details of the ISM physics: dust depletion and abundances, non-equilibrium ionisation, shocks, turbulence, etc.

\subsection{Halo-disk interaction in the Milky Way}

The Milky Way is surrounded by a large halo of hot gas which must be replenished as the gas cools. The most direct evidence comes from the detection of high-ionisation UV resonance lines and of X-ray emitting gas surrounding the Galactic disk. The X-ray halo has a luminosity of $\sim 4 \times 10^{39} \mathrm{erg} \mathrm{s}^{-1}$ and the thickness of the emission is probably a few kpc. The temperature of the X-ray emitting gas is $(1-2) \times 10^{6} \mathrm{~K}$, well below the escape temperature, and therefore it is gravitationally bound to the Galaxy. The high temperatures of the gas in the halo must be explained by energetic processes, most likely occurring in the Galactic disk (though external sources could contribute). The most likely energy source for these processes are massive OB stars.

There is also evidence that hot halo gas has cooled, as provided by the presence of the high-ionisation UV resonance lines. Some of the UV resonance lines might be produced by photoionisation of gas near $10^{4} \mathrm{~K}$, but there is good evidence that the high-ionisation lines, such as Si IV, C IV and N V are due to gas that is cooling (Savage and Sembach, 1994). For the Si IV and C IV absorption lines it was found that the ratio of the column densities from these ions is almost a constant value, implying that the ionisation state of the gas is nearly identical in different parts of the halo. Benjamin and Shapiro (1993) argued that in a galactic fountain, gas cooling from $10^{6} \mathrm{~K}$ would be opaque to its own radiation, causing it to self-ionise, so that its ionisation state is determined by the cooling process itself. They also showed that the absorption strength of $\mathrm{N} \mathrm{V}$ is reproduced by this model. Martin and Bowyer (1990) have shown that the emission from C IV and $\mathrm{O}$ III ions is consistent with being produced from a cooling Galactic fountain.

The cooling of the fountain flow, is dominated by collisional ionisation and radiative recombination as three-body recombination (the inverse process of collisional ionisation) is very unlikely to occur, because as the fountain gas, with a temperature in excess of $10^{6} \mathrm{~K}$, rises into the halo it expands and cools adiabatically. Hence, there is a reduction of the plasma temperature and density (to be of the order of $10^{-2} \mathrm{~cm}^{-3}$ ). Furthermore, the cooling timescales at considerable heights above the disk can be much smaller than the dominant microphysical processes and therefore, recombi-

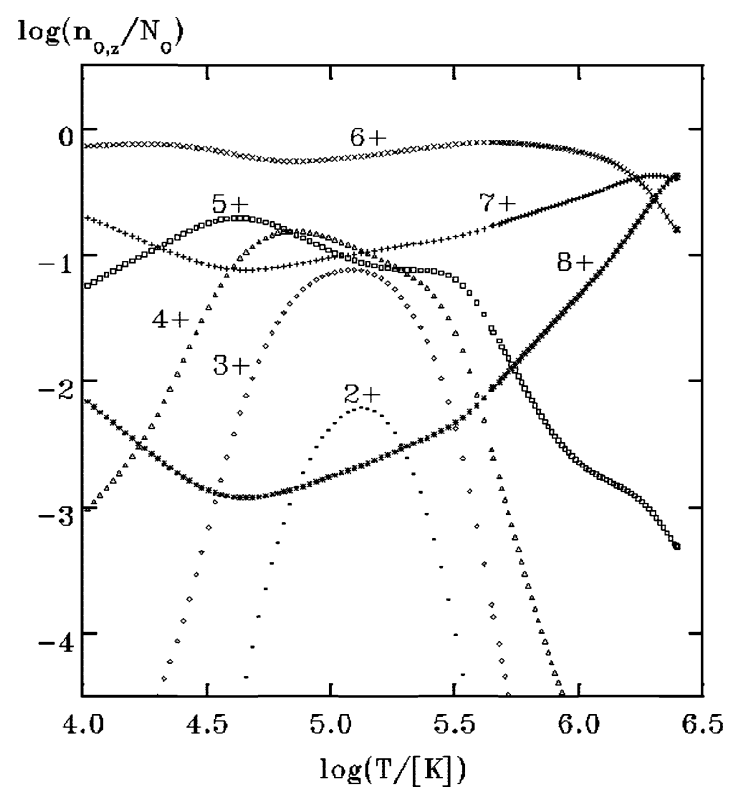

Fig. 1 Ionisation stages of oxygen in a self-consistent calculation of a galactic outflow (wind) (Breitschwerdt and Schmutzler, 1999). The initial temperature of the flow is $T=2.5 \times 10^{6} \mathrm{~K}$ 
nation of highly ionised species lags behind (Breitschwerdt and Schmutzler, 1999). An example is the O VI ion that is abundant over a large range of temperatures from $4 \times 10^{5} \mathrm{~K}$ to temperatures as low as $10^{4} \mathrm{~K}$ (Fig. 1). Thus, care should be taken while using a single ion like $\mathrm{O}^{5+}$ as a diagnostic element for plasma temperature (see also Schmutzler and Tscharnuter, 1993).

Thus, measurements of the $z$-dependence of $\mathrm{O}$ VI, $\mathrm{N} \mathrm{V}$, $\mathrm{C}$ IV and Si IV emission are of prime importance for determining whether the gas is in collisional equilibrium ionisation, in non-equilibrium ionisation, or whether other relevant heating sources maybe present. These observations are critically needed to constrain numerical models of the ISM in disk galaxies to provide further clues concerning how matter and energy are transferred within the Galaxy. This is clearly displayed in Fig. 2; adaptative mesh simulations of the dynamical evolution of the ISM (Avillez and Breitschwerdt, 2004 , in press) show that matter in the disk is concentrated in dense shells and filaments, while the halo acts as a pressure release valve for the hot $\left(T>10^{5.5} \mathrm{~K}\right)$ phase in the disk thereby controlling its volume filling factor. The upper portions of the thick ionised disk form the disk-halo interface located about $2 \mathrm{kpc}$ above and below the mid-plane. Here a large-scale fountain is set up by hot ionised gas injected from either the gas streaming out of the thick disk or directly from superbubbles in the disk underneath. The gas then escapes in a turbulent convective flow.

Radio observations can be used to map the clumpy distribution of matter in the disk, but the most important constraints will come from the study of the vertical distribution of warm gas, which is best studied with UV spectroscopy. This is also true for the high-velocity clouds (HVCs) detected by their $\mathrm{HI} 21 \mathrm{~cm}$ emission, which are surrounded by hot ionised envelopes as pointed out by new observations from the Far Ultraviolet Spectroscopic Explorer (FUSE) mission and the Space Telescope Imaging Spectrograph onboard the Hubble Space Telescope (HST/STIS). The detection of O VI, C IV and Si IV absorption indicates that many HVCs have a hot, collisionally-ionised component (Danly et al., 1992; Tripp et al., 2003). UV absorption lines provide detailed information on the physical conditions and abundances of the gas. Understanding the ionisation of such envelopes will permit us to constrain the properties of the Galactic corona and the Local Group medium. UV absorption lines are the most sensitive probes for determining the abundances (and hence their Galactic or extragalactic origin) of the HVCs (see, e.g., Richter et al., 2001). Note that the most robust specie for constraining the metallicity of HVCs is O I, since oxygen is only slightly depleted by dust grains (Moos et al., 2002) and the ionisation potential of $\mathrm{O} \mathrm{I}$ is very similar to $\mathrm{H} \mathrm{I}$. Thus, oxygen abundances based on the $\mathrm{O} \mathrm{I} / \mathrm{H}$ I ratio depend only slightly on the ionisation of the gas for substantially ionised plasmas.

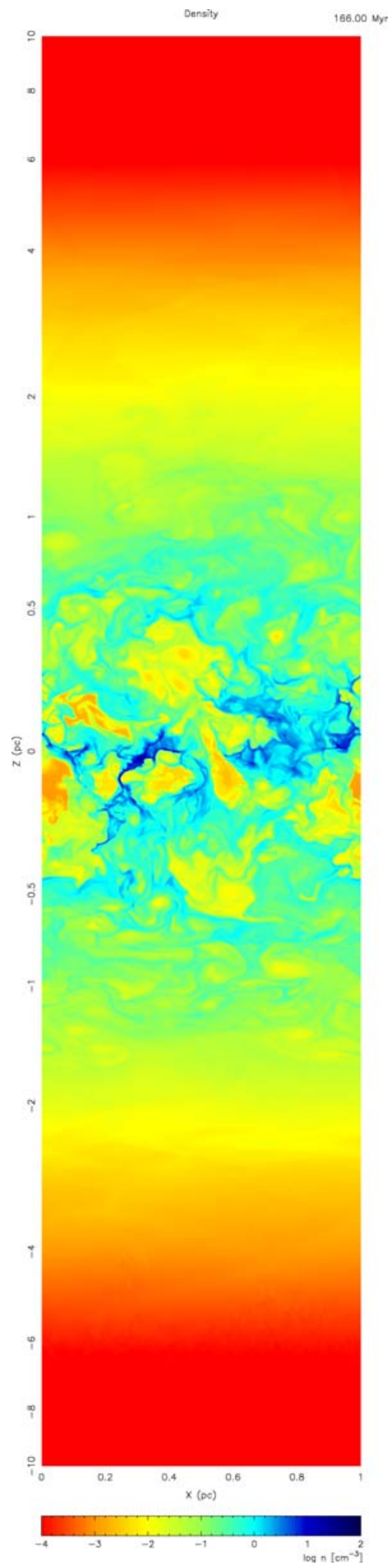

Fig. 2 Slice through the 3D data set showing the vertical (perpendicular to the mid-plane) distribution of the density at time $166 \mathrm{Myr}$. Red/blue in the colour scale refers to lowest/highest density (or highest/lowest temperature). The $z$-scale above $0.5 \mathrm{kpc}$ and below $-0.5 \mathrm{kpc}$ is shrunk (in order to fit the paper size) and thus, the distribution of the labels is not uniform (from Avillez and Breitschwerdt, in press) 


\subsection{The local ISM}

The Sun lies inside the Local Bubble, a large ionised gas bubble that extends outwards from the Sun to a neutral hydrogen column density $\log N(\mathrm{H} \mathrm{I}) \approx 19.2$ ( $\mathrm{cm}^{-2}$ units), corresponding to a geometrical size of $100-200 \mathrm{pc}$ depending on the direction from the Sun. The Local Bubble's morphology has been identified by Na I absorption, which is formed in the cold gas than surrounds the Local Bubble and determines its shape (Lallement et al., 2003). The Local Bubble is thought to be an $\mathrm{H}$ II region formed by the explosions of supernovae and the strong winds of young hot stars in the Lower Centaurus Crux subgroup of the Scorpio-Centaurus Association (Maíz-Apellániz, 2001; Berghöfer and Breitschwerdt, 2002). The temperature of the gas in the Local Bubble is estimated to be about $10^{6} \mathrm{~K}$ if most of the soft X-ray background is due to thermal emission from the Local Bubble gas. However, analysis of extreme ultraviolet emission obtained with the CHIPS satellite has not yet led to an accurate temperature or emission measure of the hot, low-density Local Bubble gas, which may be far out of collisional ionisation equilibrium.

Embedded in the Local Bubble are a number of warm gas clouds. The Sun is located inside one of these clouds called the Local Interstellar Cloud (LIC). The existence of the LIC was first suggested by Vidal-Madjar et al. (1978). The LIC was first identified by Lallement and Bertin (1992) on the basis of measured Doppler shifts of interstellar absorption lines in many directions that are consistent with a single velocity vector, implying that all of the gas in this cloud is moving with a common velocity away from the centre of the Scorpio-Centaurus Association. Analysis of interstellar UV absorption lines with the high-resolution echelle gratings in the GHRS and STIS instruments on HST enabled Redfield and Linsky (2000) to determine a temperature of the LIC gas $(7000 \pm 1000 \mathrm{~K})$ and the morphology of the LIC. The LIC centre is located in the anti-Galactic centre direction. The maximum column density through the LIC is $\log N(\mathrm{H} \mathrm{I})=$ 18.3 , the LIC's maximum dimension is about $6.8 \mathrm{pc}$, and its mass is about $0.32 M_{\odot}$. The main evidence for the Sun being located inside the LIC is that neutral helium flowing into the heliosphere, which is not influenced by the solar wind, has the same temperature and flow vector as the LIC (Bertin et al., 1993; Witte, 2004).

Slavin and Frisch (2002) computed the ionisation of many elements in the LIC taking into account UV and EUV radiation from the most important ionising source, the star $\epsilon \mathrm{CMa}$, hot white dwarfs and other stars, the diffuse UV background, and the estimated radiation from the putative conductive boundary between the warm clouds and the hot gas of the Local Bubble. More sensitive UV observations are required to study this boundary layer, if indeed it is present. Their models assumed ionisation equilibrium and realistic
$\mathrm{H}$ I column densities between the centre of the LIC and the external sources of ionising radiation. One of their models predicts the temperature, electron density, and ionisation of many elements in good agreement with observations. Dust, which is present in the LIC and other nearby warm clouds, plays an important role in cooling the gas and in depleting metals such as iron from the gas phase.

There are a number of other warm, partially ionised clouds in the solar neighbourhood, which are also located inside the Local Bubble. The so-called $G$ cloud identified by Lallement and Bertin (1992), which is situated in the Galactic centre direction, is slightly cooler than the LIC and has a somewhat different velocity vector. The closest star $\alpha$ Cen $(1.3 \mathrm{pc})$, which is located in the $G$ cloud, shows no evidence for absorption by gas at the LIC velocity even in very high signal-to-noise GHRS echelle spectra. This places an upper limit of $0.05 \mathrm{pc}$ on the thickness of the LIC in the direction of $\alpha$ Cen and a time of $<3000$ years for the Sun to leave the LIC and enter either the $G$ cloud or an unknown interface region between the LIC and the $G$ cloud.

The broad UV spectral coverage, high spectral resolution, and accurate wavelength scale of the STIS instrument allowed Redfield and Linsky (2004) to measure absorption line wavelengths, widths, and Doppler shifts for many atoms and ions including H I, D I, C II, N I, O I, Mg II, Al II, Si II and Fe II along 29 different lines of sight through warm clouds in the Local Bubble. They detected absorption at 50 different velocities along these lines of sight, indicating about 12 clouds with different velocity vectors. The observation of absorption lines from elements or ions with very different atomic weights ( 2 for deuterium compared to 56 for iron) allowed Redfield and Linsky (2004) to solve for the gas temperature and non-thermal motions (turbulence) separately for each velocity component. They found velocity components in the local ISM with temperatures as high as about $12000 \mathrm{~K}$ and a few components with temperatures below $3000 \mathrm{~K}$. The mean gas temperature is $T=6680 \pm 1490 \mathrm{~K}$, which is characteristic of warm clouds, but there are some velocity components inside the Local Bubble that could be cold clouds. In almost all cases, the non-thermal motions are far smaller than the thermal motions. The mean thermal pressure in the clouds, $P_{\mathrm{T}} / k=2280 \pm 520 \mathrm{~K} \mathrm{~cm}^{-3}$. The magnetic fields in these clouds have not yet been measured.

While STIS spectra of interstellar absorption lines formed in the local ISM have begun to reveal its secrets, we have sampled far too few lines of sight to identify the structure of the local ISM in detail. In particular, we do not yet have a good understanding of the amount of gas at different temperatures in the local ISM, nor do we have a detailed understanding of how the ionisation and temperature of the gas depends on the radiation environment and past history. Understanding the physics of the local ISM is required if we are to have any confidence in understanding the physics of the Galactic 
disk, halo, and ISM in other galaxies. Since most interstellar absorption lines are located in the UV and the absorption lines are typically narrow with multiple velocity components, a future sensitive high-resolution UV spectroscopic mission is needed to extend the preliminary work provided by the GHRS and STIS instruments on HST.

\section{The physics of accretion and outflow}

Understanding how stars form out of the contracting cores of molecular gas is a major challenge for contemporary astrophysics. Angular momentum must be conserved during gravitational contraction and magnetic flux is built up and dissipated in the process, but the underlying mechanisms are still under debate.

Solar-like protostars are an excellent laboratory for this purpose, since their pre-main sequence (PMS) phases last $\sim 100$ Myr. The collapse of low-mass protostars is subalfvénic, thus these protostars are expected to be magnetized. The detection of $\mathrm{kG}$ fields in stars as young as a few million years (Guenter et al., 1999; Johns-Krull et al., 1999) supports this assumption. In the last few years, a new paradigm has emerged to understand the basics of star formation. Protostars are assumed to be magnetized and star growth is regulated by the interaction between the stellar magnetic field and the disk. The physics of this interaction is outlined in Fig. 3. The disk-star interaction basically transforms the angular momentum of the disk (differential rotation) into plasmoids

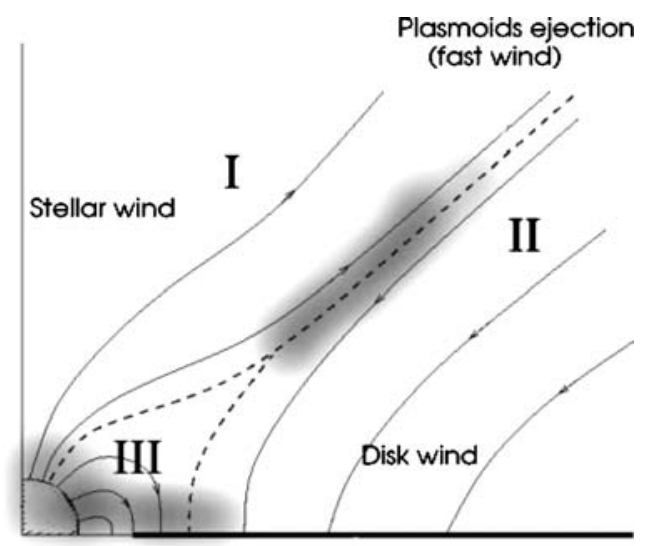

Fig. 3 The interaction between the stellar magnetic field and the disk twists the stellar field lines due to the differential rotation. The toroidal magnetic field generated out of the poloidal flux and the associated pressure tends to push the field lines outwards, inflating them, and eventually braking the magnetic link between the star and the disk (boundary between Regions I and II). Three basic regions can be defined: Region I dominated by the stellar wind, Region II dominated by the disk wind and Region III dominated by stellar magnetospheric phenomena. The dashed line traces the boundaries between this three regions. The continuous lines indicate the topology of the field and the shadowed areas represent regions where magnetic reconnection events are likely to occur, producing high-energy radiation and particles (from Gómez de Castro, 2004) that are ejected from the system. There is a current sheet that separates two distinct regions: an inner stellar outflow and an external disk flow. Magnetic flux dissipation should occur in the current layer producing the ejection of plasmoids, as well as the generation of high-energy particles (cosmic rays), $\mathrm{X}$-rays, and ultraviolet radiation.

The phenomenon is non-stationary and is controlled by two different temporal scales: the rotation period and the magnetic field diffusion timescale. Stellar rotation is a well-known parameter that controls the opening of the field lines towards high latitudes. Plasmoid ejection, however, is controlled by field diffusion which is poorly determined (see, e.g., Priest and Forbes, 2000). All models can be fitted into this basic configuration (Uzdensky, 2004).

During the last 5 years, numerical research on this interaction region has gone into outburst (see, e.g., Goodson et al., 1997, 1999). So far, most studies have analysed the interaction between a dipolar stellar magnetic field and a Kepplerian accretion disk. Numerical simulations show that the fundamental mechanism for jet formation is robust. The star-diskoutflow system is self-regulating when various initial disk densities, stellar dipolar field strengths, and primordial fields associated with the disk are tested (Matt et al., 2002), although strong stellar magnetic fields may disrupt the inner parts of the accretion disk temporarily (Kueker et al., 2003). Despite the numerical advances made so far, the real properties of the engine are poorly known because of the lack of observations to constrain the modelling. Very important open questions include the following:

1. How does the accretion flow proceed from the disk to the star? Is there any preferred accretion geometry like, for instance, funnel flows?

2. What roles do disk instabilities play in the whole accretion/outflow process?

3. What are the dominant wind acceleration processes? What are the relevant timescales for mass ejection?

4. How does this high-energy environment affect the chemical properties of the disk and planetary building?

5. How important is this mechanism when radiation pressure becomes significant as for Herbig Ae/Be stars?

Infrared and radio wavelengths cannot access this engine because the spatial scales involved are tiny $(<0.1 \mathrm{AU}$ or 0.7 mas for the nearest star-forming regions compared with ALMA's resolution of 10 mas) and the temperatures are too high (3000-300 000 K). High-resolution IR spectroscopy has indeed confirmed the presence of warm molecular gas with temperatures of $1500-3000 \mathrm{~K}$ in the innermost disk: both $\mathrm{CO}$ (fundamental and overtone) and $\mathrm{H}_{2} \mathrm{O}$ emission have been detected (see Najita et al., 2000 for a review or Carr, Tokunaga and Najita, 2004 for more recent results). Fortunately, after $1 \mathrm{Myr}$, during the classical T Tauri Star (CTTS) phase, the circumstellar extinction becomes small $\left(\mathrm{A}_{V}<1 \mathrm{mag}\right)$ 
and the engine described above can be properly tested at ultraviolet wavelengths. The UV spectral range is the richest for diagnosing astrophysical plasmas in the $3000-300000 \mathrm{~K}$ temperature range, since the resonance lines of the most abundant species are located in the UV. In addition, as the UV radiation field is strong in the circumstellar environment, fluorescence emission from the most abundant molecules $\left(\mathrm{H}_{2}\right.$, $\mathrm{CO}, \mathrm{OH}, \mathrm{CS}, \mathrm{S}_{2}, \mathrm{CO}_{2}^{+}, \mathrm{C}_{2}$, and $\mathrm{CS}$ ) is observed. As a result, a single high-resolution spectrum in the 1200-1800 $\AA$ range provides information on the molecular content, the abundance of very reactive species such as the $\mathrm{O} \mathrm{I}$, and the warm and hot gases associated with the CTTSs. The potential of UV spectroscopy for studying the physics of accretion during PMS evolution is outlined in the following section.

\subsection{UV observations of the jet engine in low-mass stars}

The engine is a small structure $(\leq 0.1 \mathrm{AU})$ with several different constituents (the accretion flow, stellar magnetosphere, winds, and inner part of the accretion disk) all radiating in the ultraviolet. The UV spectrum of the T Tauri Stars (TTSs) has a weak continuum and many strong emission lines. The continuum is significantly stronger than that observed in main sequence stars of similar spectral types ( $\mathrm{G}$ to $\mathrm{M}$ ); the so-called optical-veiling represents the low-energy tail of this excess UV emission (Hartigan et al., 1990). The underlying photosphere is barely detected, and only in warm (G-type) weak line TTSs (WTTSs) is the photospheric absorption spectrum observed. The UV continuum excess is significantly larger in CTTSs than in WTTSs, as is well illustrated in the colour (UV - V)-magnitude (V) diagram displayed in Fig. 4. Simple models of hydrogen free-free and free-bound emission

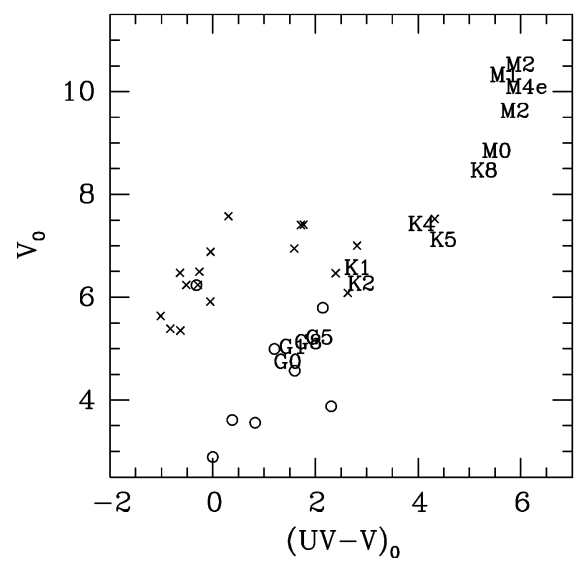

Fig. 4 The (UV - V, V) colour-magnitude diagram for the T Tauri Stars observed with the IUE satellite in the Taurus region (a distance of $140 \mathrm{pc}$ to Taurus has been assumed). The crosses represent cool TTSs (spectral types later than $\sim \mathrm{K} 3$ ) and the open circles represent warm TTSs (spectral types earlier than $\sim \mathrm{K} 3$ ). The location of the main sequence is marked by the spectral types. The stars closer to the main sequence are the WTTSs (from Gómez de Castro, 1997) added either to black bodies or to the spectra of standard stars reproduce the UV continuum reasonably well (Calvet et al., 1984; Bertout et al., 1988; Simon et al., 1990). The fits yield chromospheric-like electron temperatures of $(1-5) \times 10^{4} \mathrm{~K}$. Three different mechanisms have been proposed to generate this hot plasma and its UV continuum: (1) a dense chromosphere (Calvet et al., 1984), (2) the release of the gravitational binding energy from the infalling material (Bertout et al., 1988; Simon et al., 1990; Gullbring et al., 2000), and (3) an outflow (Ferro-Fontán and Gómez de Castro, 2003; Gómez de Castro and Ferro-Fontán, 2005). This uncertainty in the formation of the UV emission points out why high-resolution UV spectroscopy and monitoring are so crucial for understanding and constraining the physics of the engine.

\subsubsection{Signatures of accretion}

The most obvious signature of accretion is the detection of narrow red-shifted absorption components on top of the emission profiles of singly ionised species such as $\mathrm{Mg}$ II or Fe II with strong transitions in the UV at 2600 and $2800 \AA$. It is widely accepted that this absorption is produced in funnel flows: magnetic tubes connecting the inner disk to the stellar surface. However, there are no detailed maps of the funnel flows except for some attempts made in the optical range (Petrov et al., 2001; Bouvier et al., 2003). UV mapping is crucial in determining the rigidity of the flux tubes and thus the possible distortions induced by differential rotation and the magnetic diffusivity of the disk.

Funnel flows are expected to radiate over a broad range of temperature, from $3000 \mathrm{~K}$ at the disk end to some $100000 \mathrm{~K}$ at the stellar surface. Since infalling material is nearly in freefall, its kinetic energy is finally released at the stellar surface in an accretion shock that reaches temperatures of $10^{6} \mathrm{~K}$. The dominant output radiation is produced by the photoionised pre-shock infalling gas (Gómez de Castro and Lamzin, 1999; Gullbring et al., 2000). Thus, the full accretion column could be tracked by monitoring CTTSs with a high spectral resolution UV instrument, but this observation has not yet been carried out! The only UV monitoring of CTTSs was by the IUE satellite with low dispersion due to the small effective area of its $40 \mathrm{~cm}$ telescope. Nevertheless, the results are very promising as rotational modulation of the UV continuum and line fluxes were detected in DI Cep and BP Tau (Gómez de Castro and Fernández, 1996; Gómez de Castro and Franqueira, 1997a). This modulation is caused by the small size of the accretion shock, which occupies only a small fraction of the stellar surface.

An important result of these campaigns is that only 50\% of the UV continuum excess is rotationally modulated. Thus, a significant fraction of the UV excess is not produced by the accretion shock. Whether the wind or an extended magneto- 
sphere is responsible for the UV continuum excess remains a matter of debate. In fact, the coexistence of several funnel flows has been proposed to explain this fact (Muzerolle et al., 2001).

\subsubsection{Signatures of disks}

High-resolution HST/STIS spectra have revealed, for the first time, the rich UV molecular emission in CTTS. $\mathrm{H}_{2}$ fluorescence emission has now been studied in detail in the nearest CTTS, TW Hya, and the richness of the spectrum is overwhelming: Herczeg et al. (2002) detected 146 Lyman-band $\mathrm{H}_{2}$ lines, representing 19 progressions (see Fig. 5)! The observed emission is likely produced in the inner accretion disk, as are the infrared $\mathrm{CO}$ and $\mathrm{H}_{2} \mathrm{O}$ lines. The excitation of $\mathrm{H}_{2}$ can be determined from the relative line strengths by measuring self-absorption in lines originating in low-energy lower levels, or by reconstructing the Ly $\alpha$ emission line profile incident upon the warm $\mathrm{H}_{2}$ using the total flux from each fluorescing upper level and the opacity in the pumping transition. Using this diagnostic technique, Herczeg et al. (2004) estimated that the warm disk surface has a column density of $N_{\mathrm{H}_{2}}=3.2 \times 10^{18} \mathrm{~cm}^{-2}$, temperature of $T=2500 \mathrm{~K}$, and filling factor of $\mathrm{H}_{2}$ as seen from the source of the Ly $\alpha$ emission of $0.25 \pm 0.08$. The observed spectrum shows that some ground electronic state $\mathrm{H}_{2}$ levels with excitation energies as large as $3.8 \mathrm{eV}$ are pumped by Ly $\alpha$. These highly excited levels may be formed by dissociative recombination of $\mathrm{H}_{3}^{+}$, which in turn may be formed by reactions involving $\mathrm{X}$-rays and UV photons from the star. A quick inspection of the UV spectra in the IUE and HST archives shows that fluorescent $\mathrm{H}_{2}$ UV lines are observed in most of the TTSs (see also Gómez de Castro and Franqueira, 1997b; Valenti et al., 2000; Ardila et al., 2002).

The role of far-UV radiation fields and high-energy particles in the disk chemical equilibrium is now beginning to be understood. Bergin et al. (2003) showed how strong Ly $\alpha$ emission may contribute to the observed enhancement of $\mathrm{CN} / \mathrm{HCN}$ in the disk. The penetration of UV photons coming from the engine in a dusty disk could produce an important change in the chemical composition of the gas, allowing the growth of large organic molecules. In this context, UV photons photodissociating organic molecules at $\lambda>1500 \AA$ could play a key role in the chemistry of the inner regions of the disk, while those photodissociating $\mathrm{H}_{2}$ and $\mathrm{CO}$ will control the chemistry of the external layers of the disk directly exposed to the radiation from the central engine (see, e.g., Cernicharo, 2004). Ultraviolet radiation also plays a very important role in the evolution of the primary atmospheres of planetary embryos (Watson et al., 1981; Lecavelier des Etangs et al., 2004).

Strong continuum FUV emission (1300-1700 $)$ ) has been detected recently from some stars with bright molecular disks

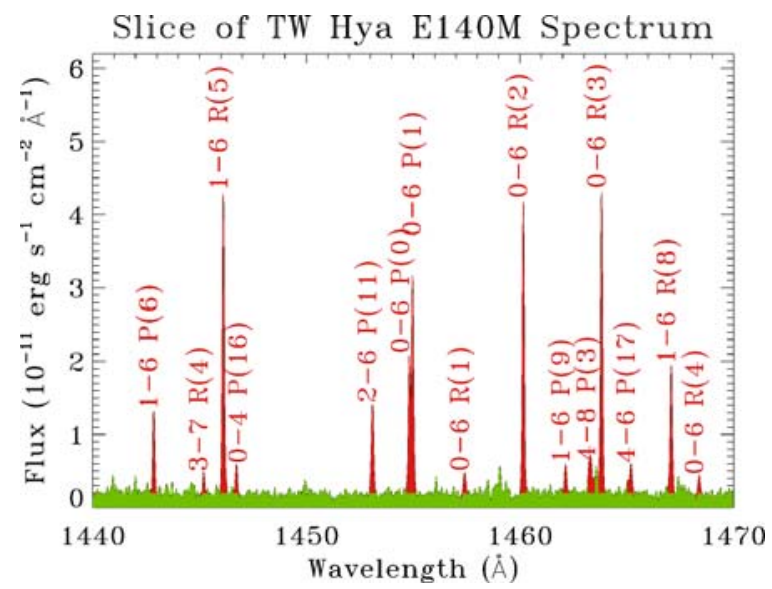

Fig. 5 A portion of the HST/STIS spectrum of the CTTS, TW Hya. The narrow $\mathrm{H}_{2}$ emission lines originate in the $B$ electronic state after being pumped by the $\mathrm{H} \mathrm{I} \mathrm{Ly} \alpha$ line (from Herczeg et al., 2002)

including GM Aur, DM Tau, and LkCa 15, together with inner disk gaps of few AUs (Bergin et al., 2004). This emission is likely due to energetic photoelectrons mixed into the molecular layer that likely indicates the existence of a very hot component in the disk. This very hot component is probably created by X-ray and high-energy particle irradiation of the disk (Glassgold et al., 2004; Gómez de Castro and Antonicci, 2005).

Spectroscopic observations of volatiles released by dust, planetesimals and comets provide an extremely powerful tool for determining the relative abundances of the vaporizing species and for studying the photochemical and physical processes acting in the inner parts of protoplanetary disks. The UV studies of $\beta$ Pic-like systems illustrate the possibilities of this spectral range for this purpose (Vidal-Madjar et al., 1994, see also, Section 3.3). The relevance of UV observations to study comets is described in detail by Brosch et al. (2005) in the Solar System chapter of this volume.

\subsubsection{Signatures of winds}

Large-scale outflows are observed as collimated jets or Herbig-Haro objects in some TTSs (see Section 3.2). However, spectroscopic signatures of winds and outflows are detected in all the TTSs. Three types of signatures have been detected in the UV:

1. The emission profiles of the $\mathrm{Mg}$ II resonance lines (2796, $2803 \AA$ ) show pronounced broad absorption in their blue wing for the 16 TTSs observed with IUE or HST (see e.g., Gómez de Castro, 1997). Blue-wing absorption is also observed in Ly $\alpha$. Terminal velocities up to $\sim 300 \mathrm{~km} \mathrm{~s}^{-1}$ are observed. Unfortunately, the interpretation of these profiles is very complex, since the Mg II and Ly $\alpha$ lines are optically thick and there is no unambiguous method 
Fig. 6 Left: Variation of the three velocity components: rotation $\left(V_{t}\right)$, radial expansion from the axis $\left(V_{r}\right)$ and axial velocity $\left(V_{z}\right)$ are represented. Top panel: $V_{z}$ and $V_{r}$ are represented with dashed and solid lines, respectively. Bottom panel: The ratio between $V_{t}$ and the Kepplerian velocity at $0.1 \mathrm{AU}$ for a solar mass star $\left(155 \mathrm{~km} \mathrm{~s}^{-1}\right)$ is plotted. Right: Disk wind kinematics as shown by the line profiles for an edge-on system. Each profile correspond to a ring of gas perpendicular to the outflow axis that is identified by its distance $(z)$ to the disk plane. Bottom panel: Line profiles with $z<5 \mathrm{AU}-$ the outflow passes from being rotationally dominated (inner broad or double peaked profile) to radial expansion dominated (double peaked profiles with peak velocity $\sim 120 \mathrm{~km} \mathrm{~s}^{-1}$. Middle panel: Outflow passes from being radial expansion dominated to axial-acceleration dominated. Top panel: Outflow is dominated by axial acceleration and the line broadening is basically thermal (from Gómez de Castro and Ferro-Fontán, 2005)

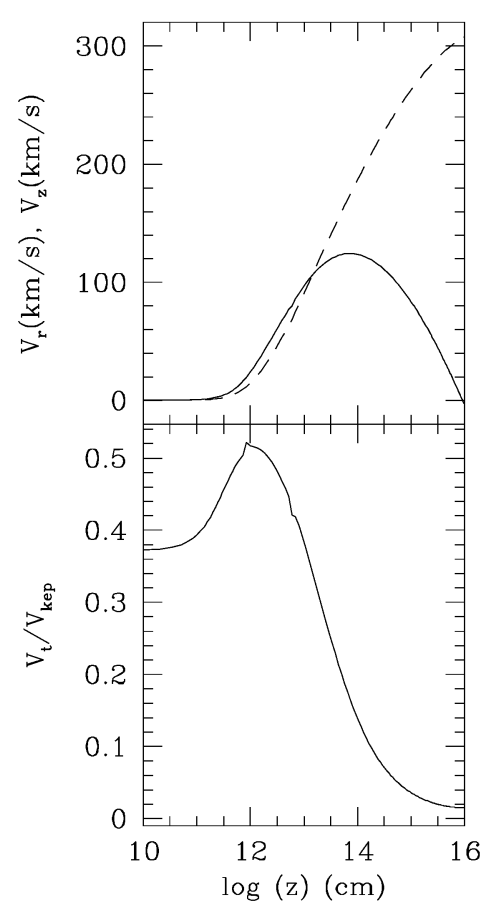

for determining the underlying blue-shifted emission and thus the true wind absorption.

2. Narrow and blue-shifted C III] $1909 \AA$ and Si III] $1892 \AA$ emission has been detected at the same velocity as the optical jets in some TTSs (Gómez de Castro and Verdugo, 2003a). This emission is produced by unresolved jets and indicates that PMS star jets are hotter than previously inferred from the optical observations in agreement with the UV observations of protostellar jets and Herbig-Haro objects (see Section 3.2).

3. Optically thin semi-forbidden lines tracing warm plasma (C II] $2325 \AA$, O II], and, most prominently, C III] and Si III]) show long blueward-shifted tails with velocities up to $-300 \mathrm{~km} \mathrm{~s}^{-1}$ as in RU Lup, together with slight shifts to the blue of the line peak (Gómez de Castro and Verdugo, 2003a).

These data provide three key pieces of information: (i) there is a broad range of temperatures in the outflows (3000 $30000 \mathrm{~K}$ ), (ii) outflows are not spherically symmetric, and (iii) their kinematics produce line broadenings/asymmetries

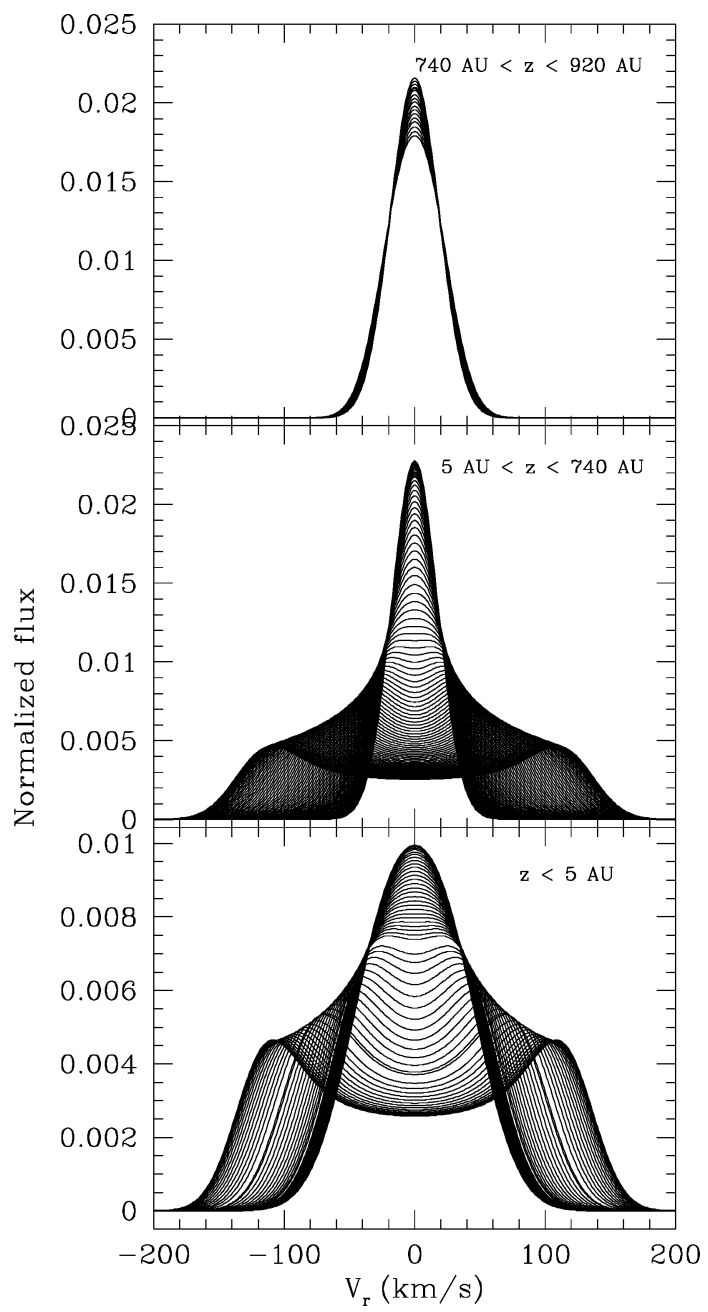

similar to the jet velocity (or terminal velocity of the outflow) in several sources.

The interpretation of the profiles requires a detailed comparison with theory, since the kinematics of MHD winds from rotating structures is very complex. Three basic motions overlap: rotation, acceleration along the axis, and radial expansion from the axis (see Fig. 6). Each kinematical component dominates at different locations in the outflow. Rotation is dominant close to the source of the outflow. Further out, radial expansion is the most significant component up to some height, $z_{0}$, above the disk. For $z>z_{0}$, the dominant kinematical component is acceleration along the disk axis, e.g., a collimated outflow or jet. For standard parameters, the base of the wind is unresolved, $z_{0}=5 \mathrm{AU}$ (see Gómez de Castro and Ferro-Fontán, 2005). Thus, the only way to track the velocity law of the wind is by a clever selection of spectral indicators based on the thermal properties of the wind.

From a theoretical point of view, three possible types of outflows can be fitted into this broad context: a stellar wind, a disk wind, and an outflow driven from the interface. Either centrifugal stresses or magnetic/thermal pressure 
Fig. $7 \mathrm{C}$ IV, C III] and C II] line profiles for the unresolved $z<12$ AU region. The profiles are plotted for inclinations $0^{\circ}$, $30^{\circ}, 45^{\circ}, 60^{\circ}$ and $90^{\circ}$ from bottom to top

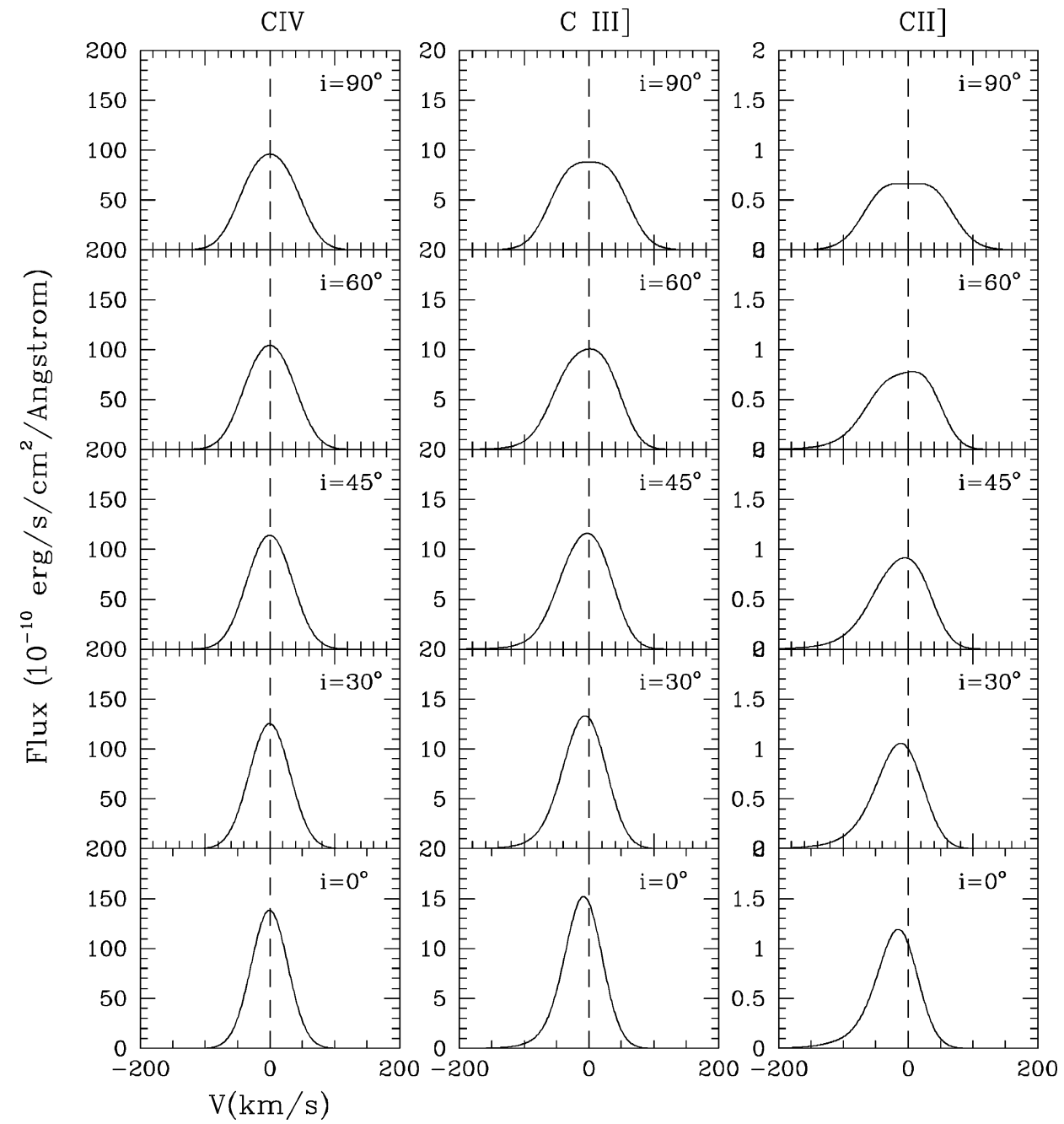

are involved in the acceleration of the outflows, but it remains unclear which mechanism is dominant, whether it is universal, and how this mechanism acts when radiation pressure becomes significant as in Herbig Ae/Be stars. Numerical simulations predict temperatures between $\sim 10000 \mathrm{~K}$ for the inner-disk winds up to $\sim 10^{6}-10^{7} \mathrm{~K}$ close to the magnetic reconnection boundary (Goodson et al., 1997). The X-rays (and the high-energy particles) produced in the reconnection areas will be redistributed towards lower energies due to the densities involved. Thus, the dominant radiative output from TTS winds is expected in the UV range, as is observed. UV line profiles calculated using a simplified warm disk wind model are shown in Fig. 7. Notice that very broad or double peaked profiles cantered at the rest wavelength of the line can be produced in the wind (not only in the accretion disk) provided that the inclination is $\sim 90^{\circ}$. These type of profiles have been observed in some TTSs such as AK Sco or RW Aur. As shown in Fig. 7, a clever selection of the UV spectral indicators helps to dissect the kinematical structure of the wind. The effect of internal wind extinction by dust lifted from the disk mid-plane can also be traced through the flux ratios of relevant lines.

Another important aspect of TTS winds is that a significant fraction of the mass outflow is ejected in a non-stationary manner. The timescales for these ejections range from a few hours (Alencar, 2001; Bouvier et al., 2003; Gómez de Castro and Verdugo, 2003b) to some 10 years (optical jets observations, see, e.g., López-Martín et al., 2003) or even to some hundreds of years (molecular gas bullets, see, e.g., Bachiller, 1996). Recent observations have established a lower limit of about $1 \mathrm{~h}$, precluding the association of flares with the few hours timescale variability in RW Aur, since the characteristic decay time of flares in active stars is some few hundreds of seconds (Gómez de Castro and Verdugo, 2003b). Several ejection timescales typically coexist in the same object. For instance, timescales of $\sim 1 \mathrm{~h}, \sim 5.5$ days and $\sim 20$ years are observed in RW Aur. Despite the wealth of valuable information that HST/STIS could have obtained to determine the kinematics and properties of these outflows, the available observations are few and not of long duration. 


\subsection{UV observations of Herbig-Haro objects and jets}

Observations of protostellar jets can provide important clues concerning the collimation mechanism: in particular, the role of episodic ejections or internal shocks in the jet, heat dissipation, and major heating sources. Also, such observations provide important clues concerning the interaction between the jet and the surrounding molecular gas, which helps to discriminate between radiation-induced (photodissociation) versus collision-induced (shocks) in the circumjet chemistry.

Since early in the IUE project, it has been known that protostellar jets and Herbig-Haro objects have a higher degree of ionisation than previously inferred from optical data (Bohm-Vitense, et al., 1982; Schwartz et al., 1985; see also Gómez de Castro and Robles, 1999 for a compilation). Highexcitation objects like $\mathrm{HH} 1$ or $\mathrm{HH} 2$ produce strong emission lines of C IV 1548, $1550 \AA$, O III] $1664 \AA$, Si III] $1892 \AA$, and C III] $1909 \AA$ A (Ortolonai and D'Odorico, 1980). However, low-excitation objects like HH43 or HH47 are characterized by the presence of the $\mathrm{H}_{2}$ Lyman band emission lines (Schwartz, 1983). $\mathrm{H}_{2}$ emission lines, which are pumped by the UV radiation generated in the internal shocks of the jet, can measure the strength of the radiation field generated in the shock and the Ly $\alpha$ line strength.

UV lines are variable and the variations of the low-ionisation species are anticorrelated with the variations of the high-ionisation species and the short-wavelength continuum. A detailed study of HH29 combining optical and UV data led Liseau et al. (1996) to propose a two-phase model with a warm component $\left(T=10^{4} \mathrm{~K}\right.$ and $\left.n_{e}=10^{3} \mathrm{~cm}^{-3}\right)$ and a hot, dense component $\left(T=10^{5} \mathrm{~K}\right.$ and $\left.n_{e}=10^{6} \mathrm{~cm}^{-3}\right)$ with a very small filling factor $(0.1-1 \%)$.

Using IUE, Bohm et al. (1987) and Lee et al. (1988) detected a variable and spatially extended short-wavelength (1300-1500 ̊) UV continuum. At the end of the IUE mission, it was believed that the most likely mechanism for its formation was continuum $\mathrm{H}_{2}$ emission formed when $\mathrm{H}_{2}$ molecules are destroyed either by photodissociation by radiation shortwards of $912 \AA$ or by collisions with low-energy thermal particles. This assumption was based on the absence of the dominant Lyman band features (at 1258, 1272, $1431,1446,1505,1547$ and $1562 \AA$ ), which are detected in such low-excitation objects as HH43 or HH47. The highresolution spectra of $\mathrm{HH} 2$ obtained with the Hopkins Ultraviolet Telescope (HUT) showed that the UV emission below $1620 \AA$ is mostly produced by $\mathrm{H}_{2}$ Lyman bands detected below $1200 \AA$ and at 1510,1580 and $1610 \AA$ (see Raymond et al., 1997). Unfortunately, this is the only highresolution spectrum of a $\mathrm{HH}$ object obtained in the far UV. HH47 was observed with HST/GHRS but the spectral coverage was tiny 1262-1298 ̊ (Curiel et al., 1995). A low-dispersion $(\simeq 1000)$ spectrum of HH47 obtained with
HST/FOS/G270H shows no significant depletion of Fe in the outflow.

The existing UV observations have left open many important questions that cannot be solved without further UV observations. It is still unclear, how the kinetic energy of the flow is damped into radiation. The non-detection of O VI emission (Raymond et al., 1997) and the simultaneous detection of strong $\mathrm{C}$ IV and $\mathrm{H}_{2}$ emission represent the strongest and most promising arguments against radiative cooling models. Another important question is how to understand the excitation mechanism of the $\mathrm{H}_{2}$ line radiation, since $\mathrm{H}_{2}$ band structure is observed in high-excitation $\mathrm{HH}$ objects. One proposed suggestion is collisional pumping of the $\mathrm{H}_{2}$ levels (Raymond et al., 1997).

\subsection{Herbig Ae/Be stars}

Herbig Ae/Be stars are intermediate-mass $\left(2-10 M_{\odot}\right)$ PMS stars. They are rather puzzling objects. Their larger masses suggest that the gravitational collapse is superalfvénic, so magnetic fields are not expected to be strong. However, Ae stars have a rich UV emission-line spectrum consistent with the presence of a chromosphere above the photosphere (Brown et al., 1996; Deleuil et al., 2005). Also, overionised species (transition region or corona-like) are observed; a marginal detection of magnetic fields has been reported for HD 104237 (Donati et al., 1997). Thus, observations point out that fields are present, at least, during the first $\sim 5 \times 10^{6}$ years of their PMS evolution.

UV-optical monitoring campaigns discovered azimuthal structures in the wind of AB Aur (Praderie et al., 1986). Subsequent optical monitoring campaigns, for example by the MUlti-SIte COntinuous Spectroscopic (MUSICOS) consortium, confirmed the presence of such azimutal structures in the wind and in the chromosphere: the rotation period of the chromospheric structures is $32 \mathrm{~h}$ (the stellar rotation period), while the rotation period of the wind (traced by the UV Mg II lines) is $45 \mathrm{~h}$ (Bohm et al., 1996; Catalá et al., 1999). Further UV observations detected clumps of very hot gas, traced by $\mathrm{N} \mathrm{V}$ emission, in the wind of $\mathrm{AB}$ Aur (Bouret et al., 1997). The generation of these azimuthal structures and the very hot clumps is often interpreted by means of a two-component wind model in some ways similar to the solar wind consisting of the following:

A "slow", dense outflow reaching terminal velocities of $\sim 300 \mathrm{~km} \mathrm{~s}^{-1}$, which produces the prominent P-Cygni profiles observed in the Ca II and Mg II[us1] lines and the broad, blue-shifted absorption observed in C Iv[uv1]. Mass-loss rates derived from semi-empirical models are a few $\times 10^{-8} M_{\odot}$ per year (Bouret and Catalá, 1998; Catalá and Kunasz, 1987). 
A "high" velocity component made by streamers of magnetically confined gas.

Since Herbig Ae/Be stars are fast rotators, gas in the streamers is forced to corotate up to the alfvén point and shocks are expected to occur between the "slow" and "fast" components. As a result, dense azimutal structures are formed in the corotating interaction regions (CIRs). The existence of a magnetic collimator is further supported by the detection of low-density Ly $\alpha$ jets in HD 163296 and HD 104237 (Devine et al., 2000; Grady et al., 2004). Magnetic field dissipation also seems the most likely source for the radiative losses in the chromosphere/wind that represent $4-8 \%$ of the stellar bolometric luminosity according to Bouret and Catalá (1998), although accretion flows may be a non-negligible energy source (Blondel et al., 1983; Bouret and Catalá, 2000; Roberge et al., 2001). Accretion, however, may not be the driver of the outflow. Radiatively driven winds are able to produce collimated outflows provided there is a magnetic field (Sakurai, 1985; Rotstein and Giménez de Castro, 1996). The ultimate source of the field remains unidentified: turbulence and rotation could set up a dynamo in the outer layers. Turbulence can be generated by stellar pulsation; radial and non-radial modes have been detected with periods from some tens of minutes to hours (see Catalá, 2003 for a review). Also, the rotational braking produced by the strong stellar wind could induce turbulent motions below the stellar surface, forcing magnetic fields into the outer stellar layers (Lignieres et al., 1996).

Future UV observations are required to characterize the winds, to study the evolution of the hot clumps (presumably shocks between the fast and slow components), and to study the physical conditions of the disks. Herbig Ae/Be stars are evolutionary precursors of the Vega-like stars, such as $\beta$ Pictoris (Vidal-Madjar et al., 1998), and thus are ideal laboratories for studying planet formation. Radio and IR observations are well suited to map the extended disk structure. Optical and IR coronographic observations with the HST have provided high-quality images of the disk structure at large (e.g., Clampin et al., 2003). However, UV spectroscopy is the most sensitive tool for determining the column density of hydrogen and the fraction of hydrogen atoms in molecular form (see, for instance, Bouret et al., 2003; Grady et al., 2005). This high sensitivity is illustrated by the very low upper limits provided by FUSE to the $\mathrm{H}_{2}$ abundance of the circumstellar disk surrounding $\beta$ Pictoris (Lecavelier des Etangs, 2001). FUSE observations have allowed to detect $\mathrm{H}_{2}$ in several disks surrounding Herbig Ae/Be stars, providing estimates of temperature, density and physical size of the emitting region (see, e.g., Roberge et al., 2001; Lecavelier des Etangs et al., 2003). Measurements of $\mathrm{H}_{2}$ abundances can provide information on the rate of $\mathrm{H}_{2}$ formation on dust grains and the strength of UV photoionising radiation.

\subsection{In summary: The potentials of the UV}

It is widely believed that infrared and radio wavelengths are the most appropriate regimes for studying the formation of stars. This perception is based on PMS stars typically being embedded in molecular clouds that strongly attenuate UV and optical radiation. This perception is true for the very early stages of star formation, but after about 1 Myr extinction generally is low and TTSs are accessible to the very powerful UV diagnostic tools. To study the evolution of TTSs and Herbig Ae/Be stars after 1 Myr is very important, because planets are formed at this time and the inner disk can be observed while the planets are forming. Also, the basic engine that regulates the formation of stars, the accretion-outflow engine, is naked at this time and can be properly observed.

Examples of some of the diagnostic capabilities of highresolution UV spectroscopy and monitoring are outlined in Figs. 8 and 9.

\section{UV line profiles can clearly disentangle accretion from out-} flow.

Figure 8 shows some UV lines in the spectrum of RY Tau. The Fe II] $2506 \AA$ line shows a broad red-shifted profile with a sharp edge at zero velocity. Since this line is pumped by Ly $\alpha$ photons, it should be formed in the accretion flow. The [O II] $2471 \AA$ line is blue-shifted and traces the wind (Gómez de Castro and Verdugo, 2005). Both lines are optically thin with no self-absorption. For comparison, the strong Mg II 2796, $2802 \AA$ lines display absorption components at the wind velocity and an extended red wing associated with the accretion flow. These two physical components could not have been disentangled from the analysis of the Mg II profile alone. In fact, the long red-shifted tail would likely have been interpreted as a signature of line saturation.

UV monitoring can be used to study the distribution of matter in the circumstellar environment.

In the solar system, there are three very different types of "flares", which are sudden increases of the high-energy radiation and high-energy particles flux: magnetic flares (magnetic reconnection events), corotating interaction regions or CIRs (shock fronts formed by the interaction between the slow and the fast component of the solar wind), and coronal mass ejections. This classification also applies to TTSs and their circumstellar environments. Highresolution UV spectroscopic monitoring is required to disentangle the possible mechanisms for flares in protostellar systems. This is feasible as shown in Fig. 9. AB Dor, a nearby 30 Myr old star, is the only young star that has been well monitored in the UV for flares. Nine events were detected during the roughly $10 \mathrm{~h}$ of monitoring with HST/GHRS! The C IV and Si IV UV line profiles produced by most of the events are narrow and red-shifted, 
Fig. 8 Profiles of some relevant UV lines in the spectrum of RY Tau. The $\mathrm{C}$ II] lines are a multiplet with many transitions producing this peculiar profile. All of the lines, except for Mg II, are forbidden or semi-forbidden (from Gómez de Castro and Verdugo, 2005)
Fig. 9 The C IV 1548 A profile of $\mathrm{AB}$ Dor during a normal stellar flare (left) and a transient feature probably associated with a CIR (right). Both events lasted several kiloseconds. The left profile is typical of three events that occurred during the short monitoring time, while the profile on the right was observed only once. Note the presence of a narrow absorption and the very broad line wings in the right panel profile (see Gómez de Castro, 2002 for more details)
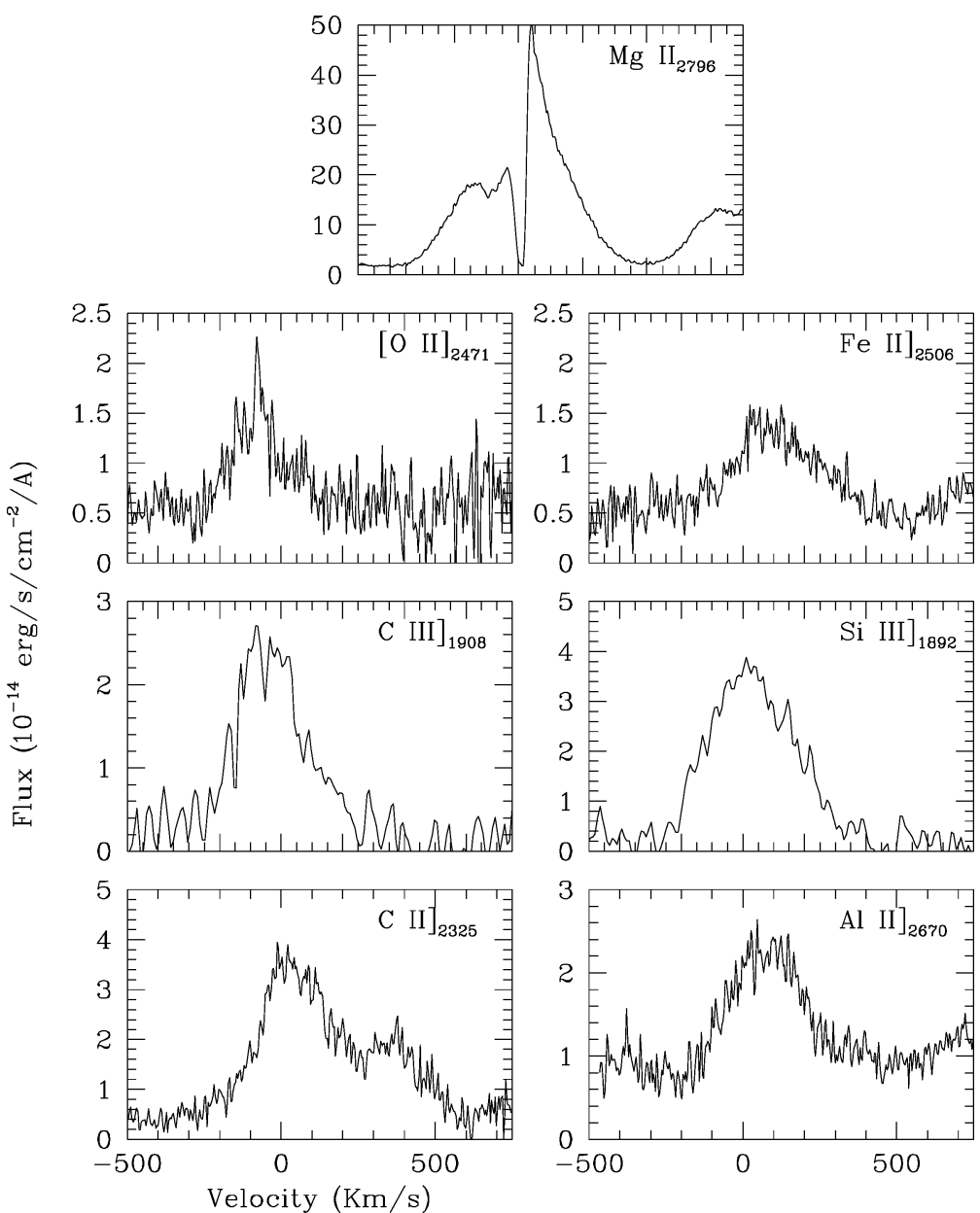

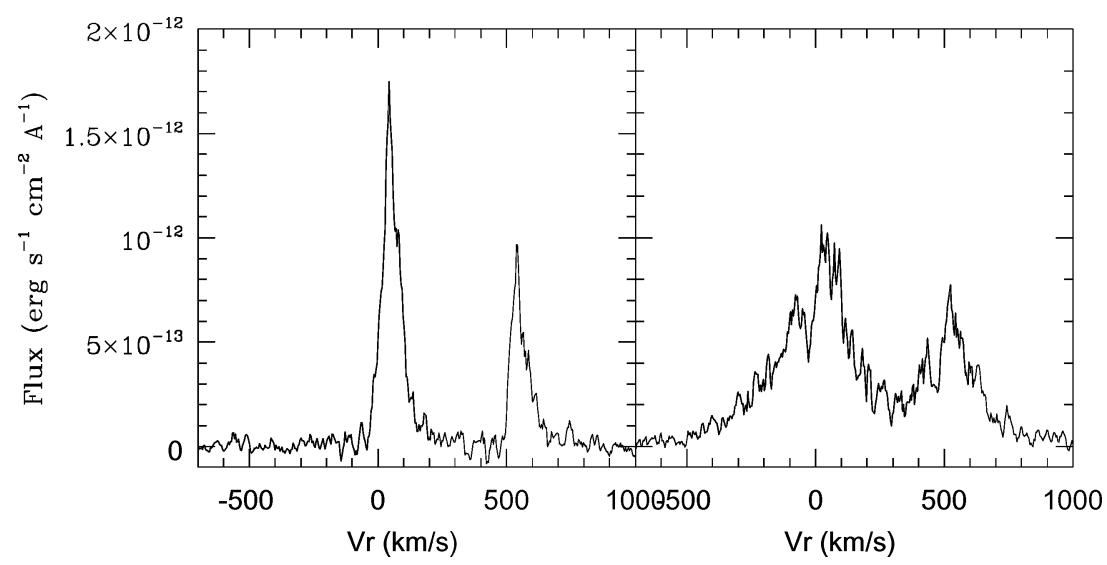

indicating hot gas falling onto the star during the flare. However, the strongest event produced a very broad profile with narrow absorption slightly blue-shifted. This profile lasted a few kiloseconds and thus the broad wings are most likely tracing the front shock of a CIR (Gómez de Castro, 2002).

In summary, IUE and HST (with its GHRS or STIS ultraviolet instruments) and FUSE have allowed us to begin to grasp the enormous potential of the UV spectral range for the

study of the physics of accretion and outflow, including the properties of the inner region of protoplanetary disks. Unfortunately, fewer than 10 TTSs were observed with spectral resolution $\sim 50000\left(6 \mathrm{~km} \mathrm{~s}^{-1}\right)$ during the lifetime of these instruments, partly because HST/STIS was not sufficiently sensitive. A UV instrument with sensitivity 50-100 times that of HST/STIS would permit observations of about 100 TTSs with magnitudes $10-13$ located within $160 \mathrm{pc}$ of the Sun. This sensitivity limit would permit observations of much fainter and more evolved WTTSs than was possible with HST/STIS. 
Fig. 10 Typical absorption spectrum of an Earth-like planet transiting in front of a solar-type star from the UV to the near infrared. We assumed the same atmospheric structure as for the Earth with, e.g., similar ozone content. Thin solid, dashed and dotted lines represent $\mathrm{H}_{2} \mathrm{O}, \mathrm{O}_{2}$ and $\mathrm{O}_{3}$ absorption. The vertical scale represents the occultation by the planet atmosphere of the stellar flux during the transit from Ehrenreich (2005)

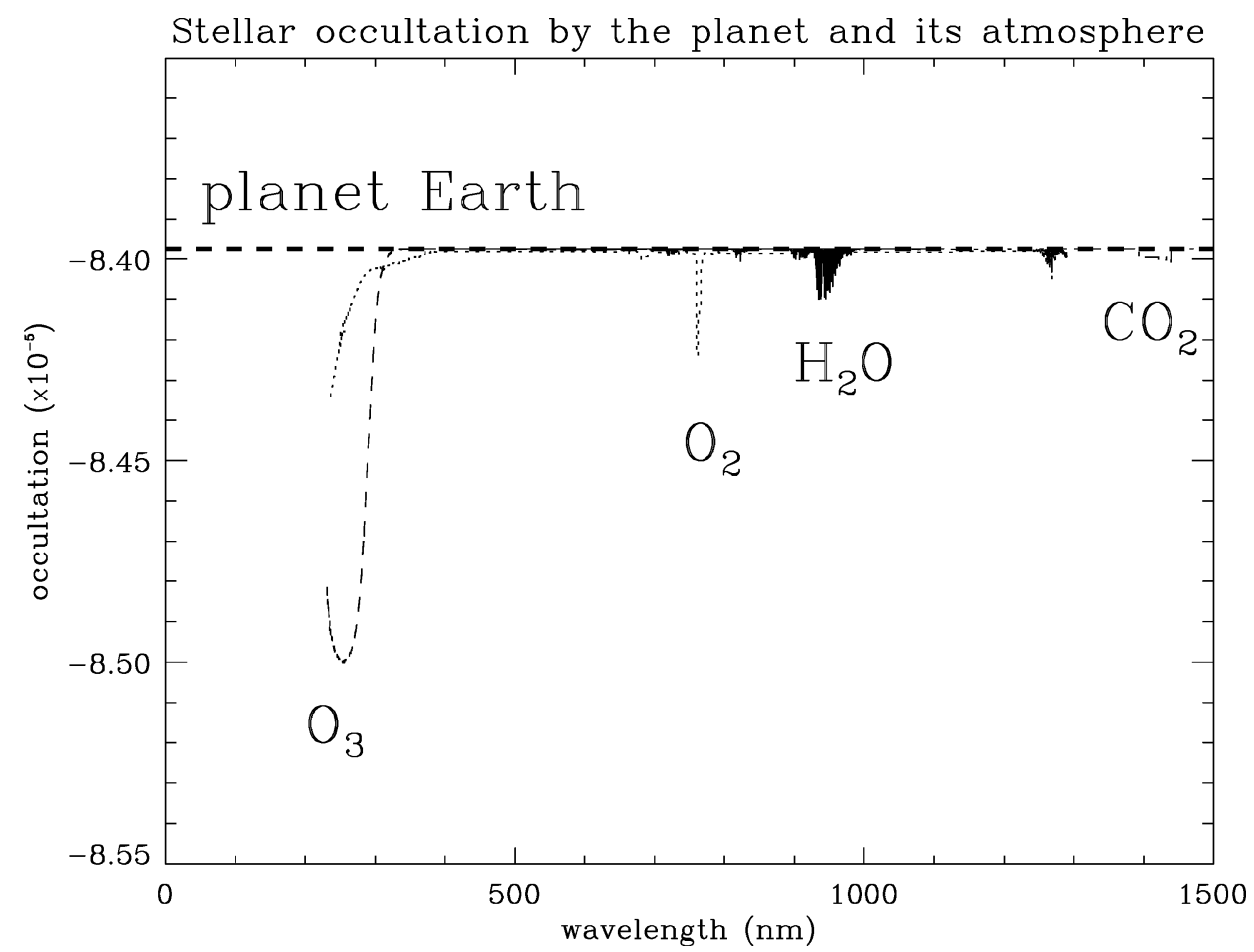

\section{Characterization of extrasolar planetary atmospheres and the search for bio-markers}

Since the mid-1990s, more than 100 extrasolar planets (hereafter called "exoplanets") have been discovered. In the coming decade, several observing programs will lead to the discovery of an extremely large number of exoplanets. To acquire a revealing picture of these new worlds, we need detailed observations of a large sample of these exoplanets to characterize planetary atmospheres well beyond the solar neighbourhood with reasonable exposure times. The observation of UV and optical absorption when an exoplanet transits its parent star is a very powerful diagnostic technique; in fact, the most powerful technique for detecting Earth-like planets because of the strong absorption of stellar UV photons by the ozone molecule in the planetary atmosphere (see Fig. 10). Near future space missions including Corot, Kepler or GAIA will lead to the discovery of a large number of exoplanets transiting their parent stars. An adequate capability for UV spectroscopic observations will be needed for detailed follow-up observations to characterize the exoplanets, their atmospheres, and their satellites.

\subsection{The physical processes controlling the formation} and evolution of exoplanets

Since the unexpected discovery of the first hot-Jupiter by Mayor and Queloz (1995), it is clear that exoplanets are an extremely diverse group. With the discovery of more than
100 exoplanets, this diversity is clearly seen in their orbital properties. We have "hot-Jupiters" with orbital periods as short as 3 days, and several "very hot-Jupiters" with orbital periods even shorter than 2 days. Less massive exoplanets have recently been discovered (Santos et al., 2004; McArthur et al., 2004; Butler et al., 2004), and the discussions on their true nature show that a large variety is now expected and certainly possible.

The same variety is also expected for the atmospheres of these exoplanets. A quick look at the atmospheric content and history of the solar system's terrestrial inner planets shows that with four planets, we find four very different possibilities: Mercury has almost no atmosphere, Mars' atmosphere is tenuous with atmospheric pressure about one-hundredth that of the Earth, and Venus is the extreme opposite with more than 90 times the atmospheric pressure of the Earth. Note that Titan, although much smaller than the Earth, also has an atmosphere of 1.5 bar and is very different from other giant planets satellites without atmospheres.

This diversity shows how difficult it is to predict what should be the content of an exoplanet's atmosphere. In the solar system, the terrestrial atmosphere is unique with abundant $\mathrm{O}_{2}$ and $\mathrm{O}_{3}$ produced by biological activity. Another important characteristic of the terrestrial atmosphere is the significant amount of water. The Earth and Titan both have much $\mathrm{N}_{2}$ in their atmospheres, but Titan has more methane and no $\mathrm{O}_{2}$. Mars and Venus have similar atmospheric composition, but their total amounts are in a ratio of more than $10^{4}$. 
Thus, there is no simple answer to the question of the expected characteristics of planets and their atmospheres. On the one hand, the solar system planets provide a first hint of the expected diversity of the exoplanets and their atmospheres. On the other hand, observations of exoplanets and the detailed characterization of their atmospheres will help us better understand the physical processes at work in the building of a planet and its atmosphere.

It is clear that the detailed processes that created the solar system planets is still a matter of debate and the impact of many processes must still be clarified. In short, we do not yet know the key physical parameters that govern the formation, evolution and fate of a given planet and its atmosphere.

How do the properties (temperature, stellar type, highenergy particles, and metallicity) of the central star alter the evolution of its planetary system? What effects do a planet's orbital parameters (orbital distance and eccentricity) have on its size, mass and potential migration during the formation process? Are there volatile-rich planets like the proposed "Ocean-planets"? How do interactions with other planets and planetesimals in their environment influence the evolution of a planet? This last question is undoubtedly related to the origin of water on the Earth. Are water-rich planets in the "habitable zone" common, rare, or exceptions?

Several processes that are believed to play key roles in building a planet can now be identified. To begin, we can look at the best known planet, the Earth. Although still controversial, it is generally accepted that the Earth's original atmosphere was accumulated simultaneously with the planet's formation. However, the heating of the atmosphere by the young Sun's UV and X-ray flux led to the hydrodynamical escape of this primary atmosphere (as is observed on HD 209458b, Vidal-Madjar et al., 2003, 2004). Tectonics, volcanism and the planet out-gasing then formed the secondary atmosphere in which we now live. Late bombardment by planetesimals in the young planetary system contributed to a large fraction of the terrestrial water but the fraction of water coming from the Earth itself and the outside contribution is still a matter of debate. Finally, life enriched the atmosphere in $\mathrm{O}_{2}$ and ozone, which are therefore considered as atmospheric bio-markers. The observation of $\mathrm{O}_{2}$ and ozone in the atmosphere of the Earth or any exoplanet can lead to the conclusion that something very particular is happening there. Something which could suggest the presence of life.

It therefore appears that we will soon discover many more exoplanets, each one likely different from the others. As soon as we will be able to characterize them in detail, there will likely be many surprises. We cannot predict what will be discovered, but this will be an unprecedented opportunity to better understand the key processes at work in the shaping of planets and, in particular, to better understand the origin of our own Earth.

\subsection{Ultraviolet observations of transiting planets}

In the coming years, many exoplanets will be discovered through transits, for example, by the Corot, Kepler and GAIA missions. In particular, GAIA will likely identify thousands of exoplanets transiting bright stars. These will be prime targets for detecting the atmospheric constituents through absorption line spectroscopy, thereby characterising the chemical and physical properties of the atmosphere, including the search for bio-markers.

Many molecules have strong electronic transitions in the UV-optical domain. This wavelength range gives access to the most important constituents of the atmospheres. In particular, bio-markers like ozone $\left(\mathrm{O}_{3}\right)$ have very strong transitions in the ultraviolet (the absorption of UV radiation by the Earth atmosphere is primarily due to $\mathrm{O}_{2}$ and $\mathrm{O}_{3}$ ). The Hartley bands of $\mathrm{O}_{3}$ are the main absorbers at 2000-3000 . $\mathrm{O}_{2}$ has strong absorptions in the range 1500-2000 $\mathrm{A}$. CO has strong bands below $1800 \AA$, and weaker Cameron bands from 1800 to $2600 \AA$. The $\mathrm{CO}^{+}$first negative bands are located in the 2100-2800 $\AA$ range. Finally, the presence of $\mathrm{CO}_{2}$ can be detected through the $\mathrm{CO}_{2}^{+}$Fox-Duffenback-Barker bands from 3000 to $4500 \AA$. We note also the important presence of O I and C II lines at 1304 and $1335 \AA$. Observation of these species can be done easily, demonstrating that these atoms and ions are present at very high altitude (several hundreds of kilometres) and providing large absorption depths.

The electronic molecular transitions are several orders of magnitude stronger than the vibrational or rotational transitions observed in the infrared or radio range. These transitions can be observed in absorption provided there is a sufficiently strong UV background source. For this reason, the observation of UV and optical absorption when a planet transits its parent star is intrinsically a more powerful diagnostic technique to characterize the atmospheres of the inner planets than infrared observations of planetary emission. This is especially true for studying small Earth-like exoplanets. It is far simpler to use the large number of photons in the stellar continuum that are absorbed in spectral lines or molecular bands by the planet's atmosphere than to attempt to cancel the huge stellar photon flux by a coronograph or interferometer to search for the faint infrared emission (thermal and scattered starlight) from the planet. In addition, the intrinsic faintness of the target sources enhances potential difficulties like confusion with exozodiacal light. Moreover, spectral observations of the atmospheres of satellites of giant planets, suspected to be numerous, are even more difficult in emission.

The first observations of the atmosphere of an exoplanet (HD 209458b, nicknamed Osiris) have been made through UV-optical spectroscopy (Charbonneau et al., 2002; VidalMadjar et al., 2003, 2004), demonstrating that it is an ideal tool for probing the atmospheric content of transiting planets. 
It is noteworthy that we already have in hand four observations of the atmosphere of an exoplanet (including the detection of oxygen). All of these present detections have been performed: (1) in space, (2) in the UV-optical wavelength range, and (3) in absorption during planetary transits. This is not a coincidence but rather a consequence of this method being the most powerful and its presenting the best trade-off of scientific result versus technical feasibility.

\subsubsection{Estimates of the expected detection rate for Earth-like exoplanets}

For a typical life-supporting Earth-like planet, the ozone layer is optically thick to UV radiation incident at a grazing angle up to an altitude of about $60 \mathrm{~km}$. This ozone layer creates an additional occultation depth of $\Delta F / F \sim 2 \times 10^{-6}$ over hundreds of Angstroms that can be compared to the $2 \times 10^{-4}$ optical depth over $1 \AA$ Aetected with HST on HD 209458. We can estimate the minimum brightness of the parent star $\left(F_{\min }\right)$ relative to the brightness of HD $209458\left(F_{\mathrm{HD} 209458}\right)$ for a detection of the ozone absorption. We have:

$$
F_{\min }=\left(\frac{\Delta F / F}{2 \times 10^{-4}}\right)^{-2}\left(\frac{\Delta \lambda}{1 \AA}\right)^{-1}\left(\frac{S}{S_{\mathrm{HST} / \mathrm{STIS}}}\right)^{-1} F_{\mathrm{HD} 209458} .
$$

With a telescope 50 times as sensitive as HST/STIS, ozone can thus be detected in Earth-like exoplanets orbiting stars brighter than $V \approx 10$ (easily identified by GAIA). This magnitude corresponds to star at a distance $d \sim 50 \mathrm{pc}$ for the latest type stars considered ( $\mathrm{K} \vee \mathrm{stars}$ ) and more than $\sim 500 \mathrm{pc}$ for the earliest stars ( $\mathrm{F}$ V stars).

With this estimate of the minimum stellar brightness needed to detect a given species, we can evaluate the number of potential targets. The number of targets with exoplanets for which we can probe the atmospheric content, $N_{\mathrm{pl}}$, is simply the total number of stars brighter than the limit $N_{*}$, multiplied by the fraction of stars having an identified transiting exoplanet at a given orbital range, $P_{\mathrm{pl}}$.

$N_{\mathrm{pl}}=N_{*} \times P_{\mathrm{pl}}$.

To evaluate the total number of stars, we must select the stellar types to be included. The usual assumption is to limit the estimates by counting only K-, G- and F-type main sequence stars. This is a conservative assumption based on the bias of the present discoveries of exoplanets. We used the conservative estimate of

$N_{*}=48000 \times 10^{0.6 *(V-10)}$.

The second term of the equation, $P_{\mathrm{pl}}$, is more difficult to quantify due to many unknowns: (i) there must be an exoplanet orbiting the star, (ii) this exoplanet must be identified, and (iii) it must be transiting the stellar disk. We made the assumption that about $25 \%$ of stars will have identified orbiting exoplanets in the near future. The probability of a transit for a given exoplanet can be estimated to be $P_{\mathrm{tr}} \approx a / R_{*}$, where $a$ is the orbital distance and $R_{*}$ is the stellar radius. For an exoplanet orbiting at $1 \mathrm{AU}$ around a solar-type star, the probability is $P_{\mathrm{tr}} \sim 0.5 \%$. If we consider the habitable zone as the most interesting orbital range, this probability increases for the smaller and more numerous stars. $P_{\text {tr }} \sim 0.5 \%$ can thus be considered as a conservative assumption for the habitable zone around solar-type stars.

Finally, we have an estimate of the number of targets with observable planets:

$N_{\mathrm{pl}} \approx 60 \times 10^{0.6 *(V-10)}$.

A combination of this last equation with the minimum brightness of the parent star needed to detect a given species, gives the size of the exoplanet sample that a telescope can analyse as a function of its sensitivity.

Similar calculations can be made for exoplanets very different from the Earth. The occultation depth is proportional to the planetary radius $\left(R_{\mathrm{p}}\right)$ multiplied by the atmospheric scale height $(h)$, and the scale height is inversely proportional to the planet's gravity $\left(g \propto M_{\mathrm{p}} / R_{\mathrm{p}}^{2}\right)$. These considerations determine that the occultation depth is related to the planet's density $\left(\rho_{\mathrm{p}}\right)$ by

$$
\Delta F / F \propto R_{\mathrm{p}}^{3} / M_{\mathrm{p}} \propto \rho_{\mathrm{p}}^{-1} .
$$

Hence, low-density exoplanets and planetary satellites will give larger absorption depths than the high-density Earth (see Fig. 11). In short, it is easier to probe the atmosphere through transit spectroscopy in the case of Ocean-planets because they are larger, or in the case of Titan-like satellites, because they are less massive than the Earth and have larger atmospheric scale heights. With an ozone layer similar to that in the Earth's atmosphere, the occultation depth of an Oceanplanet, or a Titan-like satellite, will be $\Delta F / F \sim 5 \times 10^{-6}$. The resulting number estimates of possible detections as a function of the telescope sensitivity are given in Fig. 12.

Estimates of the minimum star brightness for detecting atmospheric signatures can be translated into the number of possible detections of such atmospheric signatures in transiting exoplanets $\left(n_{\mathrm{d}}\right)$. The number of possible detections is related to the number of stars brighter than the minimum brightness $\left[n\left(F>F_{\min }\right)\right]$, the probability of finding an exoplanet around these stars $\left(P_{\mathrm{p}}\right)$, and the transit probability at a given orbital distance $\left[P_{\mathrm{t}}(d)\right] . P_{\mathrm{p}}$ is unknown and we consider $P_{\mathrm{p}}=0.25$ as a reasonable number. We also estimate the transit probability at $1 \mathrm{AU}: P_{\mathrm{t}}(1 \mathrm{AU})=0.5 \%$. For the number of stars at a given brightness, we restrict the sample to 
Fig. 11 Typical absorption spectrum of an Ocean-planet as estimated by Leger et al. (2004) when seen transiting in front of a solar-type star. We assumed the same atmospheric structure as for the Earth but rescaled the structure to the size and density as presently expected for an 6 Earth mass and 2 Earth radius volatile-rich exoplanet from Ehrenreich (2005)
Fig. 12 Plot of the number of expected detections of atmospheric signatures as a function of telescope sensitivity for Earth-like and Ocean-like planets
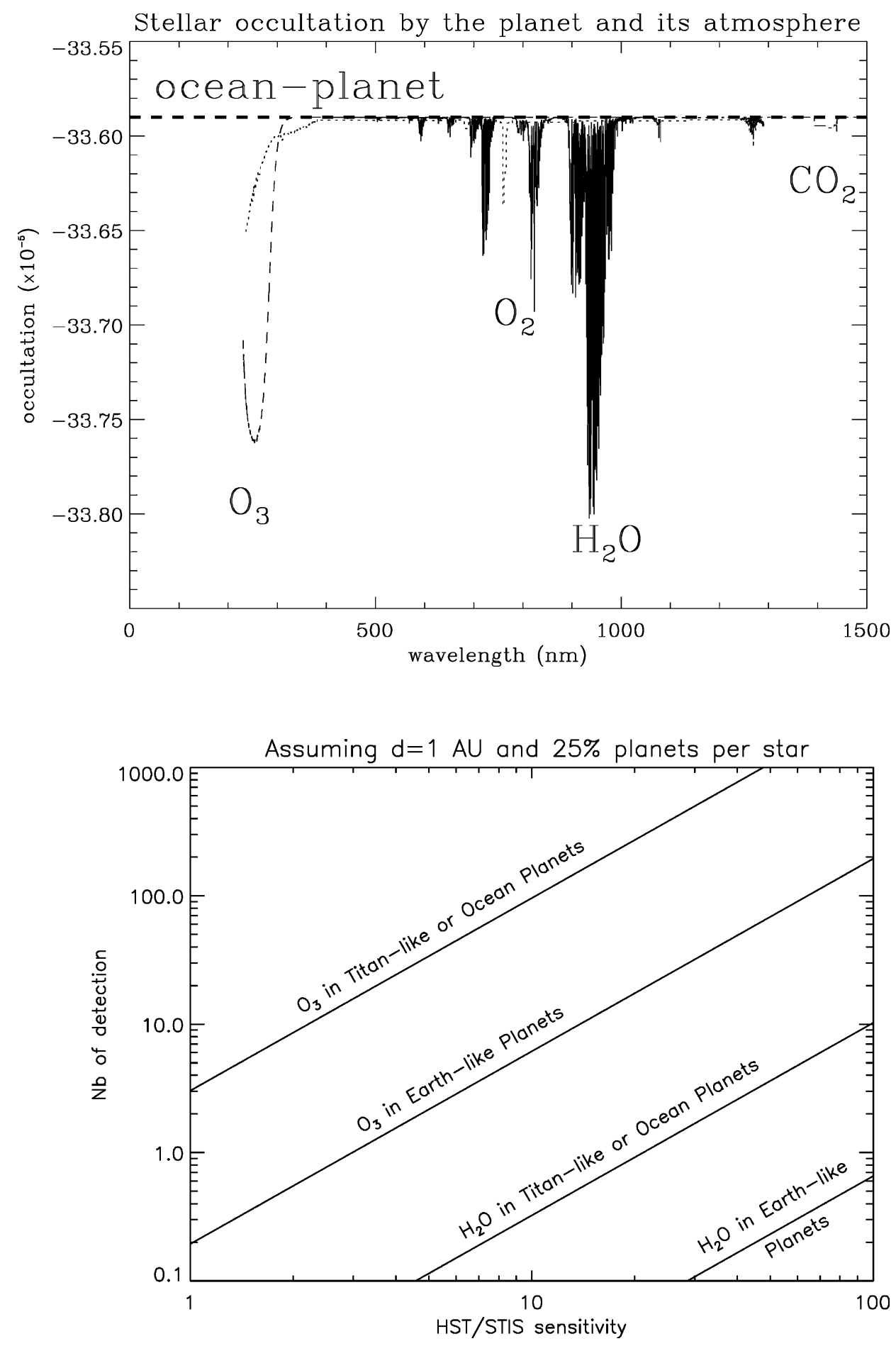

K-, G- and F-type main sequence stars. With these numbers, we plot in Fig. 12 the number of possible detections of atmospheric signatures as a function of telescope sensitivity. For a UV telescope, the sensitivity depends on the mirror size and the spectrograph efficiency. From now on, we quote the telescope sensitivity $(S)$ in units of HST/STIS sensitivity because planetary atmospheres have already been detected and studied with this instrument and, henceforth, we are not limited to a theoretical calculation that may ignore some potential difficulties. We note that a 2-m class telescope including a spectrograph with the efficiency of the COS instrument leads to a sensitivity $S \sim 20 \mathrm{HST} / \mathrm{STIS}$.

We see that about 100 exoplanets are expected to transit in front of K-, G- or F-type main sequence stars brighter than $V=10$. In conclusion, using the transit probabilities in the habitable zone, we find that the presence of bio-markers and 
other constituents in the atmospheres can be searched for in more than about 100 Earth-like exoplanets orbiting K-, Gand F-type main sequence stars. Further considerations to be taken into account are given as follows:

The effect of stellar variety. The number estimates given earlier is probably conservative. Indeed, we neglected the stellar type in the estimates and considered the real observations of HD 209458b as a benchmark. However, HD 209458 is a G-type star. A very large number of targets will be exoplanets orbiting K-type stars. For these stars, the stellar radius is smaller and the absorption depth due to the transiting planets will be larger (as observed in the case of the recently discovered exoplanet TrES-1 transiting a K0 V star (Alonso et al., 2004)).

Moreover, late-type stars are expected to have habitable zones at orbital distances smaller than $1 \mathrm{AU}$ assumed in the earlier calculation. With smaller orbital distances, the transit probability and the corresponding number of targets increase. Since the previous calculation was done for G-type stars, we expect a larger number of detections for K-type stars. The final number of possible detection of water bands should therefore be larger than is shown in Fig. 12. Water bands are likely detectable in a reasonable number of Earth-like exoplanets with a 50-100 HST/STIS sensitivity telescope.

The spatial structure of the atmosphere can be studied by time-tagged observations. Absorption spectroscopy of transiting planets can also provide spatial information on the physical and chemical properties of their atmospheres. During partial phases when the planet partially covers the observed stellar disk, time-tagged spectra provide a spatial scan of the exoplanet's atmosphere. The partial phase lasts about $10 \mathrm{~min}$ for an Earth-size exoplanet orbiting at $1 \mathrm{AU}$ from its parent star. For the closest stars $(\leq 100 \mathrm{pc})$, exposures of a few minutes will identify the atmospheric diagnostics of the most important constituents. Detailed time analysis of transit spectra can give information on the spatial distribution of atmospheric characteristics along the exoplanet's surface, for example, the difference between poles and equator or the spatial inhomogeneity of different chemical constituents.

\section{Summary: The needed capabilities}

The scientific program outlined in this article requires a broad range of instrumentation from imaging to spectroscopic capabilities.

\subsection{Imaging}

Two imaging instruments are required: a large field-of-view and a high-resolution imager.
The large field-of-view instrument will be used for two main purposes: mapping of protostellar jets and tracking flare timescales over large fields.

The high-resolution imager's primary use is to resolve the cooling structures of jets and to map protostellar disks. Coronography is required. UV observations provide the best contrast for detecting structures around young stars; for instance, a Herbig Ae/Be star is a 100 times fainter at Ly $\alpha$ than at $\mathrm{H} \alpha$. Narrow filters cantered in the most prominent UV lines like Ly $\alpha, \mathrm{C}$ IV, C III, C II, O I, He II or O VI are required.

\subsection{Spectroscopy}

Most of the science program is oriented towards spectroscopy. Two basic modes are required: high-resolution spectroscopy and medium-to-low long-slit spectroscopy.

High-resolution spectroscopy $(R \simeq 50000)$ is required for the Doppler mapping of circumstellar structures, flares, winds and disks. It is also required for detailed studies of the ISM. The spectral resolving power required to observe the atmosphere of exoplanets is not a crucial capability. We have seen that $R=\lambda / \Delta \lambda=10000$ is more than enough. Even lower resolving power, $R \lesssim 1000$, could be enough to detect the broad-band signatures of many molecules.

Note, however, that in some cases higher resolving power will resolve the thermal broadening of absorption lines in planetary exospheres (Vidal-Madjar et al., 2003). In that case, a high resolving power of $R \sim 100000$ will provide important constraints on the atmospheric structure.

Long-slit spectroscopy is required to map the spatiallyresolved jet emission, disks and circumstellar envelopes. Spectral resolution as high as 10000 is required.

Wavelength coverage. The target spectral range for the spectroscopic instruments goes from $\sim 1000 \AA$ (to include the $\mathrm{O}$ VI lines and the $\mathrm{H}_{2}$ bands) to $\sim 4000 \AA$ to have some overlap with optical telescopes and to cover most of the molecular broad-band absorption expected from exoplanet atmospheres. Extension to $10000 \AA$ would provide access to the strong water band, which is of prime interest for the search, statistics and characterization of habitable exoplanets and, consequently, for exobiology.

Sensitivity. An improvement by a factor of $20-100$ over HST/STIS capabilities will permit the study of the warm ISM beyond the Local Bubble and observe gas high in the halo towards the HVCs. It will also increase the sample of TTSs observed in the UV from some 10 to about 200 including the WTTSs providing, for the first time, an unbiased view of the accretion-outflow engine during PMS evolution. 
The sensitivity of the spectrograph should be high around prominent nebular lines like C III] $1909 \AA$ A, Si III] $1892 \AA$, and C II] $2325 \AA$.

Time-tagged observations. Accurate time information is essential. The absolute accuracy of the timescale needs to be precise to coordinate monitoring campaigns with other instruments or to study exoplanet transits. The accuracy and uniformity of the timing sets the spatial resolution for Doppler mapping. Time-tagged observations can be considered as a proxy for spatial resolution at the level of the exoplanet's size.

\subsection{Orbit}

The orbit should permit efficient observations. A long-period orbit will allow long uninterrupted observing with few Earth occultations, little airglow pollution, and minimal geocoronal emission. This will facilitate long-duration flare observations and Doppler mapping on timescales of 1 day. An L2 orbit is optimal for this purpose.

Acknowledgements This work has been supported by the European Commissions 6th Framework programme under contract number RII3Ct-2004-001566 to the OPTical Infrared CO-ordination Network for Astronomy (OPTICON). The authors are member of the Network for UltraViolet Astrophysics (NUVA): this network is defined within the OPTICON activities to structure the European astronomy. AIGdC acknowledges support by the Ministry of Science and Technology of Spain through grants AYA 2000-966, ESP2001-4637E and ESP2002-10799E. JLL acknowledges support by NASA through grant AR-09930. M.A. would like to thank Dieter Breitschwerdt for enlightening discussions on non-equilibrium ionisation.

\section{References}

Alencar, S.H.P., Johns-Krull, C.M., Basri, G.: Astrophys. J. 122, 3335 (2001)

Ardila, D.R., Basri, G., Walter, F.M., Valenti, J.A., Johns-Krull, C.M.: Astrophys. J. 566, 1100 (2002)

Avillez, M., Breitschwerdt, D.: A\&A 425, 899 (2004)

Avillez, M., Breitschwerdt, D.: A\&A 436, 585 (2005)

Bachiller, R.: Annu. Rev. Astron. Astrophys. 34, 111 (1996)

Benjamin, R., Shapiro, P.: In: E. Silver and S. Kahn (eds.), Ultraviolet and X-Ray Spectroscopy of Laboratory and Astrophysical Plasmas, Cambridge University Press, Cambridge, p. 280 (1993)

Bertout, C., Basri, G., Bouvier, J.: Astrophys. J. 330, 350 (1988)

Breitschwerdt D., Schmutzler T.: A\&A 347, 650 (1999)

Berghöfer, T.W., Breitschwerdt, D.: A\&A 390, 299 (2002)

Bertin, P., Lallement, R., Ferlet, R., Vidal-Madjar, A.: J. Geophys. Res. 98(A9), 15193 (1993)

Blondel, P.F.C., Talavera, A., Djie, H.R.E.T.A.: A\&A 268, 624 (1993)

Boehm, T., Catala, C., Donati, J.-F., Welty, A., Baudrand, J., Butler, C.J., Carter, B., Collier-Cameron, A., Czarny, J., Foing, B., Ghosh, K., Hao, J., Houdebine, E., Huang, L., Jiang, S., Nefl, J.E., Rees, D., Semel, M., Simon, T., Talavera, A., Zhai, D., Zhao, F.: Astron. Astrophys. Suppl. 120, 431 (1996)

Boehm, K.-H., Buehrke, Th., Raga, A.C., Brugel, E.W., Witt, A.N., Mundt, R.: Astrophys. J. 316, 349 (1987)
Boehm-Vitense, E., Cardelli, J.A., Nemec, J.M., Boehm, K.H.: Astrophys. J. 262, 224 (1982)

Bouret, J.-C., Catala, C.: A\&A 340, 163 (1998)

Bouret, J.-C., Catala, C.: A\&A 359, 1011 (2000)

Bouret, J.-C., Catala, C., Simon, T.: A\&A 328, 606 (1997)

Bouret, J.-C., Martin, C., Deleuil, M., Simon, T., Catala, C.: A\&A 410, 175 (2003)

Bouvier, J., Grankin, K.N., Alencar, S.H.P., Dougados, C., Fernández, M., Basri, G., Batalha, C., Guenther, E., Ibrahimov, M.A., Magakian, T.Y., Melnikov, S.Y., Pelrov, P.P., Rud, M.V., Zapatero Osorio, M.R.: A\&A 409, 169 (2003)

Calvet, N., Basri, G., Kuhi, L.V.: Astrophys. J. 277, 725 (1984)

Catala, C.: A\&SS 284, 53 (2003)

Catala, C., Kunasz, P.B.: A\&A 174, 158 (1987)

Cernicharo, J.: Astrophys. J. 608, L41 (2004)

Charbonneau, D., Brown, T.M., Noyes, R.W., Gilliland, R.L.: Astrophys. J. 568, 377 (2002)

Clampin, M., Krist, J.E., Ardila, D.R., Golimowski, D.A., Hartig, G.F., Ford, H.C., Illingworth, G.D., Bartko, F., Benétez, N., Biakeslee, J.P., Bouwens, R.J., Broadhurst, T.J., Brown, R.A., Burrows, C.J., Cheng, E.S., Cross, N.J.G., Feldman, P.D., Franx, M., Gronwall, C., Infanle, L., Kimble, R.A., Lesser, M.P., Martel, A.R., Menanteau, F., Meurer, G.R., Miley, G.K., Postman, M., Rosati, P., Sirianni, M., Sparks, W.B., Tran, H.D., Tsvetanov, Z.I., White, R.L., Zheng, W.: Astron. J. 126, 385 (2003)

Curiel, S., Raymond, J.C., Wolfire, M., Hartigan, P., Morse, J., Schwartz, R.D., Nisenson, P.: Astrophys. J. 453, 322 (1995)

Danly, L., Lockman, F.J., Meade, M.R., Savage, B.D.: ApJSS 81, 125 (1991)

Deleuil, M., Bouret, J.-C., Catala, C., Lecavelier des Etangs, A., VidalMadjar, A., Roberge, A., Feldman, P.D., Martin, C., Ferlet, R.: A\&A 429, 247 (2005)

Devine, D., Grady, C.A., Kimble, R.A., Woodgate, B., Bruhweiler, F.C., Boggess, A., Linsky, J.L., Clampin, M.: Astrophys. J. 542, L115 (2000)

Donati, J.-F., Semel, M., Carter, B.D., Rees, D.E., Collier Cameron, A.: Month. Not. R.A.S. 291, 658 (1997)

Ehrenreich, D.: PhD Thesis, Univ. de Paris VI (2005)

Ferro-Fonán, C., Gómez de Castro, A.I.: Month. Not. R.A.S. 342, 427 (2003)

Glassgold, A.E., Najita, J., Igea, J.: Astrophys. J. 615, 972 (2004)

Grady, C.A., Woodgate, B., Heap, S.R., Bowers, C., Nuth, J.A., III, Herczeg, G.J., Hill, H.G.M.: Astrophys. J. 620, 470 (2005)

Grady, C.A., Woodgate, B., Torres, C.A.O., Henning, Th., Apai, D., Rodmann, J., Wang, H.S.B., Linz, H., Williger, G.M., Brown, A., Wilkinson, E., Harper, G.M., Herczeg, G.J., Danks, A., Vieira, G.L., Malumuth, E., Collins, N.R., Hill, R.S.: Astrophys. J. 608, 809 (2004)

Gómez de Castro, A.I.: Month. Not. R.A.S. 332, 409 (2002)

Gómez de Castro, A.I.: A\&SS 292, 561 (2004)

Gómez de Castro, A.I. Verdugo, E.: APJ, submitte (2005)

Gómez de Castro, A.I., Fernández, M.: Month. Not. R.A.S. 283, 55 (1996)

Gómez de Castro, A.I., Franqueira, M.: IUE-ULDA Access Guide No. 8: T Tauri Stars, ESA Scientific Publication, ESA-SP 1205 (1997a)

Gómez de Castro, A.I., Franqueira, M.: Astrophys. J. 482, 465 (1997b)

Gómez de Castro, A.I., Ferro-Fontán, C.: MNRAS 362, 569 (2005)

Gómez de Castro, A.I., Lamzin, S.: Month. Not. R.A.S. 304, L41 (1999)

Gómez de Castro, A.I., Robles, A.: INES Access Guide No. 1: HerbigHaro Objects, ESA Scientific Publication, ESA-SP 1237 (1999)

Gómez de Castro, A.I., Verdugo, E.: Astrophys. J. 597, 443 (2003a)

Goodson, A.P., Boehm, K.-H., Winglee, R.M.: Astrophys. J. 524, 142 (1999)

Goodson, A.P., Winglee, R.M., Boehm, K.-H.: Astrophys. J. 489, 199 (1997) 
Guenther, E.W., Lehmann, H., Emerson, J.P., Staude, J.: A\&A 341, 768 (1999)

Gullbring, E., Calvet, N., Muzerolle, J., Hartmann, L.: Astrophys. J. 544, 927 (2000)

Haffner, L.M., Reynolds, R.J., Tufte, S.L., Madsen, G.J., Jaehnig, K.P., Percival, J.W.: Astrophys. J. Suppl. Ser. 149, 405 (2003)

Hartigan, P., Hartmann, L., Kenyon, S.J., Strom, S.E., Skrutskie, M.F.: Astrophys. J. 354, L25 (1990)

Heyer, M., Zweibel, E.: A\&SS 292, 9 (2004)

Herczeg, G.J., Linsky, J.L., Valenti, J.A., Johns-Krull, C.M., Wood, B.E.: Astrophys. J. 572, 310 (2002)

Herczeg, G.J., Wood, B.E., Linsky, J.L., Valenti, J.A., Johns-Krull, C.M.: Astrophys. J. 607, 369 (2004)

Johns-Krull, C.M., Valenti, J.A., Koresko, C.: Astrophys. J. 516, 900 (1999)

Kueker, M., Henning, T., Ruediger, G.: Astrophys. J. 589, 397 (2003)

Kulkarni, S., Heiles, C.: Galactic and Extragalactic Radio Astronomy, Springer-Verlag, Berlin and New York, p. 95 (1988)

Lallement, R., Bertin, P.: A\&A 266, 479 (1992)

Lallement, R., Welsh, B.Y., Vergely, J.L., Crifo, F., Sfeir, D.: A\&A 411, 447 (2003)

Lauroesch, J.T., Meyer, D.M., Blades, J.C.: Astrophys. J. 543, L43, (2000)

Lecavelier des Etangs, A., Deleuil, M., Vidal-Madjar, A., Roberge, A., LePetit, F., et al.: A\&A 407, 935 (2003)

Lecavelier des Etangs, A., Vidal-Madjar, A., McConnell, J.C., Hébrard, G.: A\&A 418, L1 (2004)

Lecavelier des Etangs, A., Vidal-Madjar, A., Roberge, A., Feldman, P.D., Deleuil, M., et al.: Nature 412, 706 (2001)

Lee, M.G., Bohm, K.H., Temple, S.D., Raga, A.C., Mateo, M.L., Brugel, E.W., Mundt, R.: Astron. J. 96, 1690 (1988)

Lignieres, F., Catala, C., Mangeney, A.: A\&A 314, 465 (1996)

Liseau, R., Huldtgren, M., Fridlund, C.V.M., Cameron, M.: A\&A 306 255 (1996)

López-Martin, L., Cabrit, S., Dougados, C.: A\&A 405, L1 (2003)

Maíz-Apellániz, J.: Astrophys. J. 560, L83 (2001)

Martin, C., Bowyer, S.: Astrophys. J. 350, 242 (1990)

Matt, S., Goodson, A.P., Winglee, R.M., Boehm, K.-H.: Astrophys. J. 574, 232 (2002)

McKee, C.F., Ostricker, J.P.: Astrophys. J. 218, 148 (1977)

Moos, H.W., Sembach, K.R., Vidal-Madjar, A., York, D.G., Friedman, S.D., Hébrard, G., Kruk, J.W., Lehner, N., Lemoine, M., Sonneborn, G., Wood, B.E., Ake, T.B., André, M., Blair, W.P., Chayer, P., Gry, C., Dupree, A.K., Ferlet, R., Feldman, P.D., Green, J.C., Howk, J.C., Hutchings, J.B., Jenkins, E.B., Linsky, J.L., Murphy, E.M., Oegerle, W.R., Oliveira, C., Roth, K., Sahnow, D.J., Savage, B.D., Shull, J.M., Tripp, T.M., Weller, E.J., Welsh, B.Y.,
Wilkinson, E., Woodgate, B.E.: Astrophys. J. Suppl. Ser. 140, 3 (2002)

Muzerolle, J., Calvet, N., Hartmann, L.: Astrophys. J. 550, 944 (2001)

Ortolani, S., D’Odorico, S.: A\&A 83, L8 (1980)

Petrov, P.P., Gahm, G.F., Gameiro, J.F., Duemmler, R., Ilyin, I.V., et al.: A\&A 369, 993 (2001)

Praderie, F., Catala, C., Simon, T., Boesgaard, A.M.: Astrophys. J. 303, 311 (1986)

Priest, E., Forbes, T.: Magnetic reconnection: MHD Theory and Applications, Cambridge University Press, New York (2000)

Raymond, J.C., Blair, W.P., Long, K.S.: Astrophys. J. 489, 314 (1997)

Redfield, S., Linsky, J.L.: Astrophys. J. 534, 825 (2000)

Redfield, S., Linsky, J.L.: Astrophys. J. 613, 1004 (2004)

Reynolds, R.J., Chaudhary, V., Madsen, G.J., Haffner, L.M.: Astron. J. 129, 927 (2005)

Richter, P., Savage, B.D., Wakker, B.P., Sembach, K.R., Kalberla, P.M.W.: Astrophys. J. 549, 281 (2001)

Roberge, A., Lecavelier des Etangs, A., Grady, C.A., Vidal-Madjar, A., Bouret, J.-C., et al.: Astrophys. J. 551, L97 (2001)

Rotstein, N, Gimenez de Castro, C.G.: Astrophys. J. 464, 859 (1996)

Sakurai, T.: A\&A 152, 121 (1985)

Savage, B.D., Sembach, K.R.: Astrophys. J. 434, 145 (1994)

Schmutzler, T., Tscharnuter, W.M.: A\&A 273, 318 (1993)

Schwartz, R.D.: Annu. Rev. Astron. Astrophys. 21, 209 (1983)

Schwartz, R.D., Dopita, M.A., Cohen, M.: Astron. J. 90, 1820 (1985)

Shapiro, P.R., Moore, R.T.: Astrophys. J. 207, 460 (1976)

Simon, T., Vrba, F.J., Herbst, W.: Astron. J. 100, 1957 (1990)

Slavin, J.D., Frisch, P.C.: Astrophys. J. 565, 364 (2002)

Tripp, T.M., Wakker, B.P., Jenkins, E.B. Bowers, C.W., Danks, A.C., Green, R.F., Heap, S.R., Joseph, C.L., Kaiser, M.E., Linsky, J.L., Woodgate, B.E.: Astron. J. 125, 3122 (2003)

Valenti, J.A., Johns-Krull, C.M., Linsky, J.L.: Astrophys. J. Suppl. Ser. 129, 399 (2000)

Vidal-Madjar, A., Lagrange-Henri, A.-M., Feldman, P.D., Beust, H., Lissauer, J.J., Deleuil, M., Ferlet, R., Gry, C., Hobbs, L.M., McGrath, M.A., McPhate, J.B., Moos, H.W.: A\&A 290, 245 (1994)

Vidal-Madjar, A., Laurent, C., Bruston, P., Audouze, J.: Astrophys. J. 223, 589 (1978)

Vidal-Madjar, A., Lecavelier des Etangs, A., Désert, J.-M., Ballester, G.E., Ferlet, R., Hébrard, G., Mayor, M.: Nature 422, 143 (2003)

Vidal-Madjar, A., Lecavelier des Etangs, A., Ferlet, R.: Planet. Space Sci. 46, 629 (1998)

Vidal-Madjar, A., Désert, J.-M., Lecavelier des Etangs, A., Hébrard, G., Ballester, G.E., Ehrenreich, D., Ferlet, R., McConnell, J.C., Mayor, M., Parkinson, C.D.: Astrophys. J. 604, L69 (2004)

Watson, A.J., Donahue, T.M., Walker, J.C.G.: Icarus 48, 150 (1981)

Witte, M.: A\&A 426, 835 (2004) 